\title{
Taxonomy of Some Recently Described Species in the Family Enterobacteriaceae
}

\author{
R. SAKAZAKI, K. TAMURA, R. JOHNSON, ${ }^{1}$ AND R. R. COLWELL \\ National Institute of Health of Japan 284, Kamiosaki-Chajamaru, Shinagawa-Ku Tokyo, Japan, and \\ Department of Microbiology, University of Maryland, College Park, Maryland 20742
}

The taxonomic positions of several recently described species, Levinea malonatica, Levinea amalonatica, Citrobacter diversus, and Enterobacter agglomerans, were investigated by numerical analysis. A set of 141 strains, for which a total of 240 characters was recorded, was analyzed and also compared with representatives of a set of 384 strains of bacteria, examined in an earlier study, representing genera within the family Enterobacteriaceae. Three clusters of Citrobacter spp. were observed, Citrobacter freundii, Citrobacter spp., and Levinea amalonatica, with strains received as Citrobacter diversus and Levinea malonatica clustering with the Citrobacter spp. Citrobacter intermedius was concluded to be synonymous with $C$. freundii. L. malonatica, from the results of this study, was included in the species $C$. diversus. Hydrogen sulfide-positive strains of Escherichia coli were not judged to warrant separate species status. Klebsiella aerogenes, Klebsiella pneumoniae, Klebsiella oxytoca, and Klebsiella edwardsii were found to be highly related (similarity values $>90 \%$ ). It is proposed that these species be merged into a single species, Klebsiella pneumoniae.

In a recently published study, 384 strains of bacteria representing genera within the family Enterobacteriaceae and the genus Yersinia were subjected to numerical analysis (38). Thirty-three phenetic clusters were distinguished, and these, for the most part, corresponded to the established species within the family Enterobacteriaceae. Useful information concerning taxonomic relationships among the Enterobacteriaceae was obtained. However, some members of the Enterobacteriaceae, including Erwinia, and the more recently described species Levinea malonatica, Levinea amalonatica, Citrobacter diversus, Enterobacter agglomerans, and Erwinia, were not represented. Therefore, an additional 141 strains of Enterobacteriaceae were examined, and the results were compared with those of the previous study.

\section{MATERIALS AND METHODS}

Bacterial strains. A list of the 141 strains included in the analysis is given in Table 1 . The name Citrobacter intermedius has been kept through this paper, although Sedlak (58) in the 8th edition of Bergey's Manual of Determinative Bacteriology has applied the name Citrobacter intermedius to $C$. diversus (L. malonatica) and L. amalonatica.

\footnotetext{
${ }^{1}$ Present address: Department of Bacteriology, American Type Culture Collection, 12301 Parklawn Dr., Rockville, Md. 20852.
}

Morphology. Strains were subcultured onto Trypticase soy agar (BBL) and incubated at $30 \mathrm{C}$ for 19 to $24 \mathrm{~h}$. Cell form was examined by staining with Loeffler methylene blue. An India ink, wet-film method was used to detect capsules. Flagella were observed using Leifson's stain (11) and by electron microscopy. Gram reaction was determined using the Hucker modification of the Gram staining procedure (11). Colonial morphology was described from cultures grown on Trypticase soy agar (BBL) for 18 to $24 \mathrm{~h}$ at $30 \mathrm{C}$. Growth in nutrient broth was determined using heart infusion broth (Difco), followed by incubation at $30 \mathrm{C}$ for up to 5 days.

Pigmentation. Pigmentation was recorded from observation of growth on the media of King et al. (41), yeast extract-mannitol agar (27), and heart infusion agar (Difco) containing either $0.2 \%$ (wt/vol) DL-phenylalanine or $0.5 \%(\mathrm{wt} / \mathrm{vol})$ tyrosine.

Physiology and resistance. Tolerance to sodium chloride was detected in nutrient broth (Difco) containing $0,0.5,3.0,5.0,7.0$, or $10 \%(\mathrm{wt} / \mathrm{vol}) \mathrm{NaCl}$. Temperature growth ranges ( 0 to $44.5 \mathrm{C}$ ) for cultures were determined in nutrient broth (Difco). Range of $\mathrm{pH}$ (4.0 to 10.0) for growth was tested on nutrient agar (Difco) in which the $\mathrm{pH}$ was adjusted, after autoclaving, with tris(hydroxymethyl)aminomethane and citrate phosphate buffer. Hemolysis was detected on heart infusion agar (Difco) containing $2 \%$ washed sheep red blood cells. Antibiotic sensitivities were tested on heart infusion agar (Difco) containing one of the following antibiotics: penicillin $(2.5 \mathrm{U}$ and $10 \mathrm{U})$, dihydrostreptomycin $(2.5 \mu \mathrm{g}$, $10 \mu \mathrm{g}$, and $30 \mu \mathrm{g})$, chloromycetin $(2.5 \mu \mathrm{g}, 10 \mu \mathrm{g}$, and $30 \mu \mathrm{g})$, erythromycin $(15 \mu \mathrm{g})$, kanamycin $(30 \mu \mathrm{g})$, aureomycin $(30 \mu \mathrm{g})$, novobiocin (10 $\mu \mathrm{g}$ and $30 \mu \mathrm{g})$, 
TABLE 1. Strains assigned to clusters in Fig. 1 and 2

\begin{tabular}{|c|c|c|c|}
\hline Cluster identification & $\begin{array}{l}\text { Strain computer } \\
\text { no. }\end{array}$ & Strain received as: & Source $^{a}$ \\
\hline \multirow[t]{3}{*}{ Proteus mirabilis } & 3 & P. mirabilis 71 & R. Sakazaki \\
\hline & 4 & P. mirabilis 72 & R. Sakazaki \\
\hline & 5 & P. mirabilis 79 & R. Sakazaki \\
\hline \multirow[t]{3}{*}{ Proteus morganii } & 6 & P. morganii 2191 & R. Sakazaki \\
\hline & 7 & P. morganii 2192 & R. Sakazaki \\
\hline & 8 & P. morganii 2193 & R. Sakazaki \\
\hline \multirow[t]{3}{*}{ Edwardsiella tarda } & 9 & E. tarda 1223 & R. Sakazaki \\
\hline & 10 & E. tarda 2000 & R. Sakazaki \\
\hline & 11 & E. tarda 1343 & R. Sakazaki \\
\hline \multirow[t]{3}{*}{ Salmonella typhi } & 12 & S. typhi 113 & R. Sakazaki \\
\hline & 13 & S. typhi 111 & R. Sakazaki \\
\hline & 14 & S. typhi 112 & R. Sakazaki \\
\hline \multirow[t]{3}{*}{ Proteus rettgeri } & 15 & $P$. rettgeri 103 & R. Sakazaki \\
\hline & 16 & $P$. rettgeri 101 & R. Sakazaki \\
\hline & 17 & $P$. rettgeri 102 & R. Sakazaki \\
\hline \multirow[t]{15}{*}{ Escherichia coli $\mathrm{H}_{2} \mathrm{~S}+$} & 19 & E. coli $\mathrm{H}_{2} \mathrm{~S}+\mathrm{N} 114$ & R. Sakazaki \\
\hline & 20 & $E$. coli $\mathrm{H}_{2} \mathrm{~S}+\mathrm{N} 111$ & R. Sakazaki \\
\hline & 21 & $E$. coli $\mathrm{H}_{2} \mathrm{~S}+\mathrm{N} 110$ & R. Sakazaki \\
\hline & 22 & E. coli $\mathrm{H}_{2} \mathrm{~S}+\mathrm{N} 46$ & R. Sakazaki \\
\hline & 23 & E. coli $\mathrm{H}_{2} \mathrm{~S}+\mathrm{N} 76$ & R. Sakazaki \\
\hline & 24 & E. coli $\mathrm{H}_{2} \mathrm{~S}+\mathrm{N} 73$ & R. Sakazaki \\
\hline & 25 & E. coli $\mathrm{H}_{2} \mathrm{~S}+\mathrm{N} 103$ & R. Sakazaki \\
\hline & 26 & E. coli $\mathrm{H}_{2} \mathrm{~S}+\mathrm{N} 68$ & R. Sakazaki \\
\hline & 27 & E. coli $\mathrm{H}_{2} \mathrm{~S}+\mathrm{N} 99$ & R. Sakazaki \\
\hline & 28 & $E$. coli $\mathrm{H}_{2} \mathrm{~S}+\mathrm{N} 63$ & R. Sakazaki \\
\hline & 29 & $E$. coli $\mathrm{H}_{2} \mathrm{~S}+\mathrm{N} 21$ & R. Sakazaki \\
\hline & 30 & E. coli $\mathrm{H}_{2} \mathrm{~S}+\mathrm{N} 5$ & R. Sakazaki \\
\hline & 31 & $E$. coli $\mathrm{H}_{2} \mathrm{~S}+\mathrm{N} 2$ & R. Sakazaki \\
\hline & 32 & E. coli $\mathrm{H}_{2} \mathrm{~S}+\mathrm{N} 28$ & R. Sakazaki \\
\hline & 33 & E. coli $1326-70$ & R. Sakazaki \\
\hline \multirow[t]{4}{*}{ Escherichia coli } & 34 & E. coli $1441-70$ & R. Sakazaki \\
\hline & 35 & E. coli $1324-70$ & R. Sakazaki \\
\hline & 36 & E. coli $1120-70$ & R. Sakazaki \\
\hline & 37 & E. coli $1233-70$ & R. Sakazaki \\
\hline \multirow[t]{4}{*}{ Hafnia alvei } & 38 & H. alvei 13 & R. Sakazaki \\
\hline & 39 & H. alvei 7081 & R. Sakazaki \\
\hline & 40 & H. alvei 5621 & R. Sakazaki \\
\hline & 41 & H. alvei $887-61$ & R. Sakazaki \\
\hline \multirow[t]{9}{*}{ Citrobacter freundii/intermedius } & 42 & C. freundii 577-67 & R. Sakazaki \\
\hline & 43 & C. freundii 423-71 & R. Sakazaki \\
\hline & 44 & C. freundii 511-71 & R. Sakazaki \\
\hline & 45 & C. intermedius 5 & R. Sakazaki \\
\hline & 46 & C. intermedius 1 & R. Sakazaki \\
\hline & 47 & C. intermedius 4 & R. Sakazaki \\
\hline & 48 & C. intermedius 2 & R. Sakazaki \\
\hline & 49 & C. freundii $624-71$ & R. Sakazaki \\
\hline & 50 & C. intermedius 3 & R. Sakazaki \\
\hline \multirow[t]{9}{*}{ Salmonella spp. } & 51 & C. freundii $624-70$ & R. Sakazaki \\
\hline & 52 & S. enteritidis & R. Sakazaki \\
\hline & 53 & S. meleagridis & R. Sakazaki \\
\hline & 54 & S. derby & R. Sakazaki \\
\hline & 55 & S. infantis & R. Sakazaki \\
\hline & 56 & S. livingstone & R. Sakazaki \\
\hline & 57 & S. arizonae 888 & R. Sakazaki \\
\hline & 58 & Unknown & R. Sakazaki \\
\hline & 59 & S. arizonae 114 & R. Sakazaki \\
\hline \multirow[t]{3}{*}{ Levinea amalonatica } & 60 & L. amalonatica 25406 & ATCC \\
\hline & 61 & L. amalonatica 25405 & ATCC \\
\hline & 62 & L. amalonatica 25407 & ATCC \\
\hline \multirow{2}{*}{$\begin{array}{l}\text { Citrobacter diversus/Levinea ma- } \\
\text { lonatica }\end{array}$} & 63 & L. malonatica 25409 & ATCC \\
\hline & 64 & L. malonatica $527-71$ & R. Sakazaki \\
\hline
\end{tabular}


Table 1-Continued

\begin{tabular}{|c|c|c|c|}
\hline Cluster identification & $\begin{array}{c}\text { Strain computer } \\
\text { no. }\end{array}$ & Strain received as: & Source $^{a}$ \\
\hline & 65 & C. diversus $1066-70$ & NCDC \\
\hline & 66 & C. diversus $2572-70$ & NCDC \\
\hline & 67 & C. diversus $2737-70$ & NCDC \\
\hline & 68 & C. diversus $3613-63$ & NCDC \\
\hline & 69 & C. diversus 2292-70 & NCDC \\
\hline & 70 & C. diversus $2393-70$ & NCDC \\
\hline & 71 & C. diversus 2524-70 & NCDC \\
\hline & 72 & L. malonatica 25408 & ATCC \\
\hline & 73 & L. malonatica 25410 & ATCC \\
\hline & 74 & L. malonatica 528-71 & R. Sakazaki \\
\hline & 75 & C. diversus $1381-70$ & NCDC \\
\hline & 76 & C. diversus $2469-70$ & NCDC \\
\hline \multirow[t]{8}{*}{ Enterobacter cloacae } & 78 & E. cloacae 102-71 & R. Sakazaki \\
\hline & 79 & E. cloacae 116 & R. Sakazaki \\
\hline & 80 & E. cloacae 101-71 & R. Sakazaki \\
\hline & 81 & E. cloacae 1679 & R. Sakazaki \\
\hline & 82 & E. cloacae 2011 & R. Sakazaki \\
\hline & 83 & Unidentified 216 & R. Sakazaki \\
\hline & 84 & Unidentified 221 & R. Sakazaki \\
\hline & 85 & Unidentified 215 & R. Sakazaki \\
\hline \multirow[t]{7}{*}{ Enterobacter agglomerans } & 86 & E. agglomerans 184-71 & NCDC \\
\hline & 87 & E. agglomerans $6-71$ & NCDC \\
\hline & 88 & E. agglomerans $1379-71$ & NCDC \\
\hline & 89 & E. agglomerans $185-71$ & NCDC \\
\hline & 90 & E. agglomerans $459-71$ & NCDC \\
\hline & 91 & E. agglomerans $219-71$ & NCDC \\
\hline & 92 & Unidentified 213 & R. Sakazaki \\
\hline \multirow[t]{9}{*}{ Klebsiella pneumoniae } & 94 & K. pneumoniae 72 & R. Sakazaki \\
\hline & 95 & $K$. pneumoniae 65 & R. Sakazaki \\
\hline & 96 & K. oxytoca 13183 & ATCC \\
\hline & 97 & K. oxytoca $833-64$ & R. Sakazaki \\
\hline & 98 & K. oxytoca 13182 & ATCC \\
\hline & 99 & K. oxytoca $1296-63$ & R. Sakazaki \\
\hline & 100 & K. oxytoca $1210-70$ & R. Sakazaki \\
\hline & 101 & $K$. edwardsii 9496 & NCTC \\
\hline & 102 & K. edwardsii 5054 & NCTC \\
\hline \multirow[t]{10}{*}{ Klebsiella rhinoscleromatis } & 111 & $K$. rhinoscleromatis i-501-56 & Orskov \\
\hline & 112 & K. rhinoscleromatis $\mathrm{i}-1-50$ & Orskov \\
\hline & 113 & K. rhinoscleromatis i-236-53 & Orskov \\
\hline & 114 & K. rhinoscleromatis i-255-53 & Orskov \\
\hline & 115 & $K$. rhinoscleromatis i- $6-50$ & Orskov \\
\hline & 116 & $K$. rhinoscleromatis i-9-50 & Orskov \\
\hline & 117 & K. rhinoscleromatis $\mathrm{i}-235-53$ & Orskov \\
\hline & 118 & K. rhinoscleromatis i-256-53 & Orskov \\
\hline & 119 & K. rhinoscleromatis i-234-53 & Orskov \\
\hline & 120 & $K$. rhinoscleromatis $\mathrm{i}-11-50$ & Ørskov \\
\hline \multirow[t]{5}{*}{ Klebsiella ozaenae } & 121 & K. ozaenae $9-72$ & R. Sakazaki \\
\hline & 122 & K. ozaenae 160 & R. Sakazaki \\
\hline & 123 & K. ozaenae 130 & R. Sakazaki \\
\hline & 124 & K. ozaenae 2015-62 & R. Sakazaki \\
\hline & 125 & K. ozaenae $15-72$ & R. Sakazaki \\
\hline \multirow[t]{3}{*}{ Enterobacter liquefaciens } & 129 & E. liquefaciens 2214 & R. Sakazaki \\
\hline & 130 & E. liquefaciens 1689 & R. Sakazaki \\
\hline & 131 & E. liquefaciens 1493 & R. Sakazaki \\
\hline \multirow[t]{5}{*}{ Serratia marcescens } & 132 & S. marcescens $2121-68$ & R. Sakazaki \\
\hline & 133 & S. marcescens 16 & R. Sakazaki \\
\hline & 134 & S. marcescens $2187-68$ & R. Sakazaki \\
\hline & 135 & S. marcescens $2249-68$ & R. Sakazaki \\
\hline & 136 & S. marcescens 14 & R. Sakazaki \\
\hline \multirow[t]{2}{*}{ Strains not clustered } & 1 & E. liquefaciens 222 & R. Sakazaki \\
\hline & 2 & Unknown coliform 224 & R. Sakazaki \\
\hline
\end{tabular}


TABLE 1-Continued

\begin{tabular}{|c|c|c|c|}
\hline Cluster identification & $\begin{array}{c}\text { Strain computer } \\
\text { no. }\end{array}$ & Strain received as: & Source $^{a}$ \\
\hline & 18 & $E$. coli $\mathrm{H}_{2} \mathrm{~S}+\mathrm{N} 59$ & R. Sakazaki \\
\hline & 77 & C. diversus $2555-70$ & $\mathrm{NCDC}$ \\
\hline & 93 & Erwinia carotovora 979 & $\mathrm{CCH}$ \\
\hline & 103 & Unknown coliform 222 & R. Sakazaki \\
\hline & 104 & Erwinia carotovora 630 & CCM \\
\hline & 105 & Enterobacter agglomerans $258-71$ & NCDC \\
\hline & 106 & Erwinia carotovora 1016 & $\mathrm{CCM}$ \\
\hline & 107 & Erwinia carotovora 1012 & CCM \\
\hline & 108 & Erwinia herbicola Aj 2189 & Tokyo Univ. \\
\hline & 109 & Erwinia herbicola Aj 2190 & Tokyo Univ. \\
\hline & 110 & Enterobacter agglomerans 5379-71 & NCDC \\
\hline & 126 & Erwinia herbicola Aj 2671 & Tokyo Univ. \\
\hline & 127 & Enterobacter liquefaciens 225 & R. Sakazaki \\
\hline & 128 & Hafnia alvei 114 & R. Sakazaki \\
\hline & 137 & Enterobacter agglomerans $3737-71$ & NCDC \\
\hline & 138 & Enterobacter agglomerans 1741-71 & $\mathrm{NCDC}$ \\
\hline & 139 & Erwinia amylovora 1020 & $\mathrm{CCM}$ \\
\hline & 140 & Erwinia amylovora 1017 & CCM \\
\hline & 141 & Enterobacter agglomerans 1743-71 & NCDC \\
\hline
\end{tabular}

${ }^{a}$ Key to sources given in footnote $a$, Table 3 .

terramycin $(2.5 \mu \mathrm{g}$ and $30 \mu \mathrm{g})$, tetracycline $(2.5 \mu \mathrm{g}$, $10 \mu \mathrm{g}$, and $30 \mu \mathrm{g}$ ), or polymyxin B (50 U). Sensitivity to Vibriostat $0 / 129$ was detected by placing several crystals of the reagent on freshly inoculated heart infusion agar (Difco).

Biochemical reactions. The mode of glucose metabolism was tested using the oxidation-fermentation medium of Hugh and Leifson (BBL). Production of acid and gas from other carbohydrates or carbohydrate derivatives was detected in peptone water (Difco) containing Andrade indicator and 1\% (wt/ vol) filter-sterilized carbohydrate.

Other reactions included in the analyses were as follows: production of dextran or levan on nutrient agar (Difco) containing 5\% (wt/vol) sucrose; methyl red and Voges-Proskauer in MRVP broth (BBL); oxidase by the methods of Kovacs (44) and Gaby and Free (29); catalase activity was detected in heart infusion broth (Difco) after incubation for $24 \mathrm{~h}$, followed by the addition of several drops of $20 \%$ hydrogen peroxide; phosphatase by the method of BairdParker (2); reduction of nitrate and nitrite determined in peptone water (Difco) containing $0.1 \%$ potassium nitrate or potassium nitrite; gelatin liquefaction on Kohn charcoal-gelatin (42) on agar slant cultures incubated for 10 days at $30 \mathrm{C}$; agar digestion by the method of Colwell and Wiebe (11); action on litmus milk; growth and hydrolysis on skim milk agar made from heart infusion agar (Difco) containing 1\% skim milk; ammonia production from Trypticase peptone water (BBL); hydrogen sulfide production from semisolid medium containing $2 \%$ thiotone (BBL), $0.5 \% \mathrm{NaCl}$, and $0.02 \%$ ferric ammonium citrate; hydrogen sulfide from cystine detected in a semisolid medium containing $1 \%$ Trypticase, $0.02 \%$ L-cystine, $0.5 \% \mathrm{NaCl}$, and $0.02 \%$ ferric ammonium citrate; Christensen urease (BBL); Rustigan urease (56); indole production determined by the method of Macfarlane et al. (49); hydrolysis of Tweens 20, 40,
60 , and 80 on Sierra medium (59); corn oil lipase detected on the corn oil medium of Hugo and Beveridge (36); hydrolysis of tributyrin on heart infusion agar containing tributyrin; citrate utilization on Koser (43), Simmons (60), and Christensen media (9); phenylalanine deaminase detected on phenylalanine agar of Ewing et al. (23); amino acid decarboxylases in Møller medium (Difco) containing arginine, lysine, ornithine, or glutamic acid; starch hydrolysis on heart infusion agar (Difco) containing $0.2 \%$ starch; esculin hydrolysis by the method of Vaughn and Levine (65); serum digestion on Loeffler inspissated serum incubated for 10 days at $30 \mathrm{C}$; eggwhite digestion on Dorset egg slants (BBL) incubated for 10 days at $30 \mathrm{C}$; cooked meat digestion tested in cooked meat medium (Difco) incubated for 10 days at $30 \mathrm{C}$; beta-galactosidase detected by the method of Le Minor and Ben Hamida (48); deoxyribonuclease on deoxyribonuclease medium (BBL); and utilization of the following organic compounds as sole carbon sources determined in Simmons agar base (60) (organic esters were in the form of the sodium salt)-acetate, citrate, malonate, lactate, fumarate, succinate, pyruvate, D-malate, L-malate, formate, tartrate, oxalate, glutarate, DL-glycerate, D-glucuronate, $\alpha$-ketoglutarate, aconitate, enanthate, propionate, butyrate, valerate, isobutyrate, isovalerate, caproate, caprylate, caprate, benzoate, DL- $\beta$-hydroxybutyrate, para-hydroxybenzoate, paraaminobenzoate, gamma-aminobutyrate, aminobutyrate, phenylacetate, quinate, hippurate, alginate, glutamate, aspartate, $N$-acetylglucosamine, ethanol, propanol, $\beta$-alanine, spermine, betain, sarcosine, $\mathrm{L}-\alpha$ alanine, D- $\alpha$-alanine, threonine, glycine, arginine, ornithine, lysine, leucine, serine, histidine, phenylalanine, tryptophan, tyrosine, citrulline, valine, isoleucine, and proline. Other reactions included in the analyses were as follows: growth, slime production, and oxidation of gluconate were recorded on Haynes 
medium (34); chitin hydrolysis was determined by the method of Benton (4); ammonia from arginine was detected in arginine broth of Niven et al. (52); acid from cystine using Hinshaw medium (35); hydrolysis of tyrosine on the medium of Gordon and Smith (32); xanthine hydrolysis on xanthine agar of Gordon and Mihm (31); tryptophan deaminase tested by the method of Singer and Volcani (61); utilization of malonate in Ewing medium (23); hydrolysis of alginate on Davis and Ewing medium (15); pectate hydrolysis on Starr medium (63); decomposition of phenylpropionic acid by the method of d'Alessandro and Comes (1); utilization of $1 \%$ (vol/vol) putrescine as sole carbon source detected in ammonium salt medium; growth on nutrient agar (Difco) containing one of the following inhibitory substances: $0.1 \%$ (wt/vol) Tergitol 7, triphenyltetrazolium chloride $(0.001 \%$ and $0.01 \%$ [wt/vol] $)$, cetrimide $(0.1 \%)$, potassium tellurite $(0.3 \%$ [wt/vol] $)$, or sodium azide $(0.05 \%$ [wt/vol]). Growth on SS agar (Difco), brilliant green agar (BBL), deoxycholate citrate agar (Difco), TCBS (Difco), and in tetrathionate (Difco) and selenite (Difco) broths was also recorded. Utilization of the organic acids D-tartrate, mucate, and citrate was detected on the medium of Kauffmann and Petersen (40).

Computation of data. A total of 240 characters was investigated. The data were coded in binary notation, using 1 for positive and 0 for negative. Noncomparable or missing characters were coded as 3 and were not included in subsequent calculations. All characters were assigned equal weight, and subsequent computations were carried out on an IBM $370 / 165$ electronic computer using a numerical taxonomy program developed by one of us (R.R.C.). Similarities between every pair of strains were calculated using both the coefficient of Jaccard, $S_{J}$, in which negative matches are excluded from the calculations, and by the simple matching coefficient, $\mathrm{S}_{\mathrm{M}}$, which includes negative matches in the similarity computation. Subsequent clustering was derived by single linkage analysis using the $S_{\mathrm{J}}$ coefficient (62).

\section{RESULTS}

The results of the initial computations are shown in Fig. 1 and 2. The 141 strains formed a large group at the 59\% phenon level. Within this large group, 19 clusters were distinguished at varying levels of similarity. Twenty-one strains did not fall into any of the 19 clusters, although some were atypical in only a few characters. At least seven strains showed little similarity to any of the other strains, indicating wide divergence from the main group.

Thirteen of the 19 clusters were readily identified as belonging to the following species: Serratia marcescens, Serratia liquefaciens, Klebsiella ozaenae, Klebsiella rhinoscleromatis, Enterobacter cloacae, Salmonella species, Hafnia alvei, Escherichia coli, Proteus rettgeri, Salmonella typhi, Edwardsiella tarda, Proteus morganii, and Proteus mirabilis. The remaining six clusters provided interesting taxonomic information and were analyzed further.

Fourteen strains of $E$. coli capable of producing hydrogen sulfide when grown on peptone iron agar joined with a non- $\mathrm{H}_{2} \mathrm{~S}$ producer at $81 \%$ similarity. Thirteen of the strains, all capable of producing hydrogen sulfide, joined at $85 \%$. Members of this cluster shared highest similarities with other strains of $E$. coli which did not produce hydrogen sulfide. Characters useful for differentiating the two clusters are shown in Table 2.

Eight strains received as Citrobacter freundii or $C$. intermedius formed a separate cluster at the $86 \%$ similarity level and were joined by a single strain of $C$. freundii (strain 42 in Fig. 1) at $84 \%$. Four of the nine strains were labeled $C$. freundii, and the remaining five strains were labeled $C$. intermedius. The strains did not separate into two subgroups on the basis of their specific epithets, although one character, production of hydrogen sulfide on peptone iron agar, was positive for the $C$. freundii strains and negative for $C$. intermedius strains.

Fourteen strains received as Levinea malonatica and Citrobacter diversus formed a cluster at $89 \%$ similarity. Here again there was no evidence of subgroups corresponding to the nomenclatural designation, although strains labeled $L$. malonatica, unlike those of $C$. diversus, were able to hydrolyze esculin and were unable to utilize citrate, when grown in the medium of Kauffmann and Petersen, or produce acid from cystine. All 14 strains formed a cluster which joined at the $84 \%$ phenon level with three strains of $L$. amalonatica. These latter strains, which included the type strain (ATCC 25405, our strain 61 ), formed a highly homogenous group at $94 \%$ and differed from the L. malonatica/C. diversus cluster in eight characters, among which were included ability to produce acid from adonitol and to grow in Møller potassium cyanide medium and on propanol and para-hydroxybenzoate as sole carbon sources. Propionate was not utilized as a sole carbon source nor was malonate reduced in Ewing medium.

Seven strains, six of which were received as Enterobacter agglomerans, and the other, as an unidentified coliform, grouped together at the $79 \%$ similarity level. All strains were motile and five of the seven were aerogenic. All were capable of growth in Møller potassium cyanide medium; however, this group showed considerable variation in a great majority of characters recorded.

Finally, nine strains received as Klebsiella 


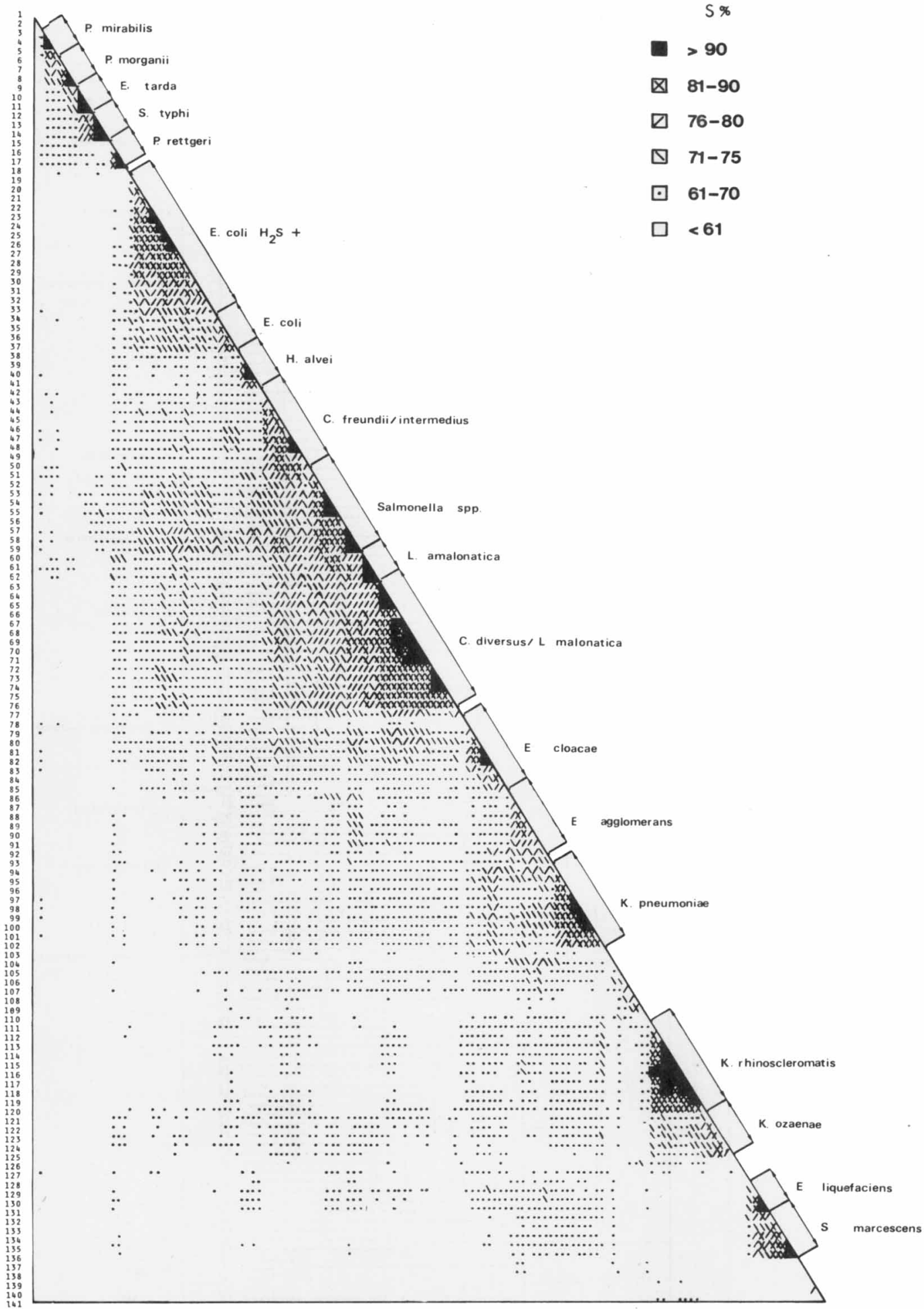

Fig. 1. Similarity (S) value matrix prepared from the numerical taxonomy analysis of the 141 strains included in the study. 


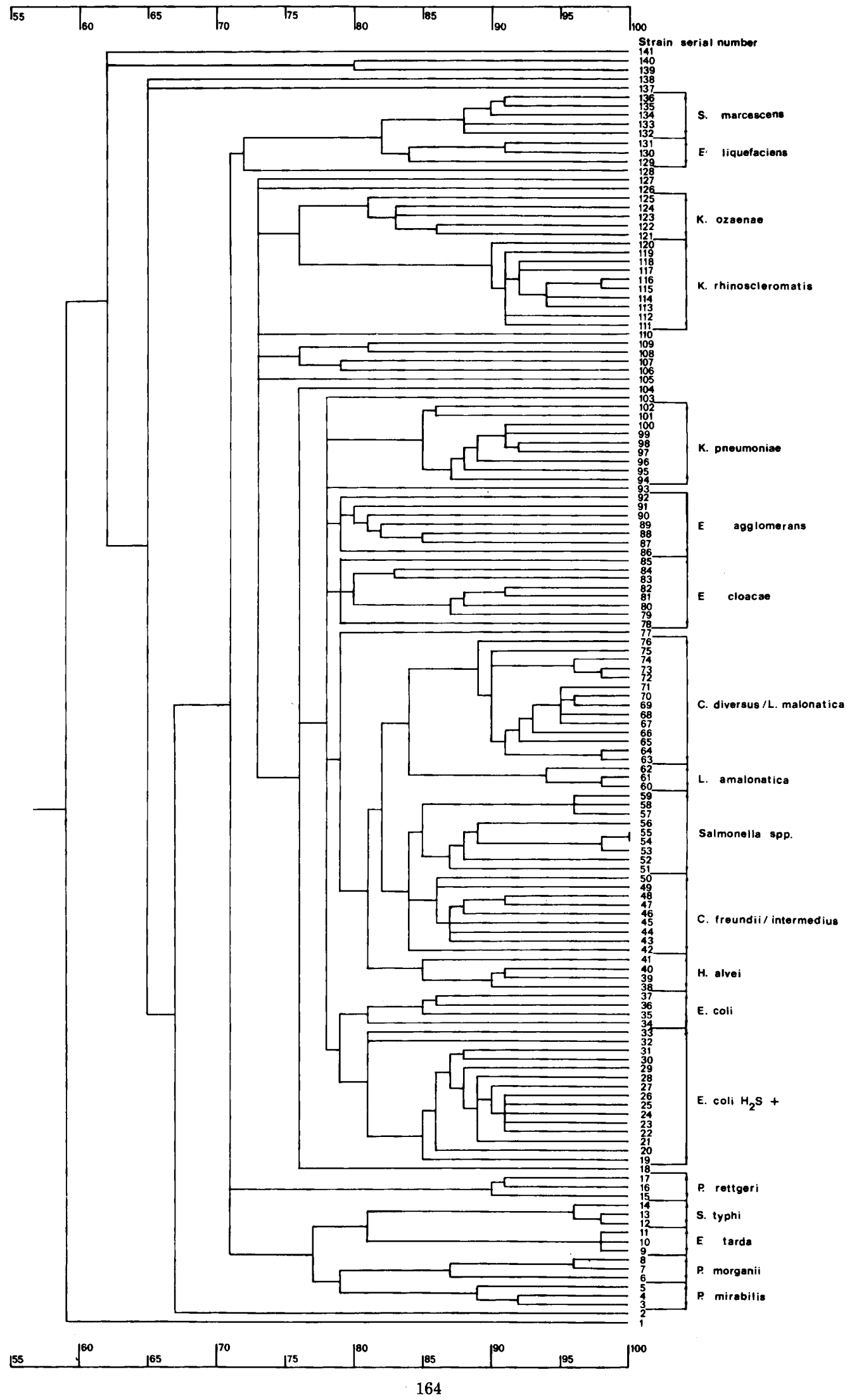


TABLE 2. Characters distinguishing hydrogen sulfide-positive strains of Escherichia coli from the more typical hydrogen sulfide-negative strains ${ }^{a}$

\begin{tabular}{|c|c|c|c|}
\hline Character & $\begin{array}{c}\mathrm{H}_{2} \mathrm{~S}-\text { posi- } \\
\text { tive } \\
\text { strains } \\
(14)^{b}\end{array}$ & $\begin{array}{c}\mathrm{H}_{2} \mathrm{~S}-n e g- \\
\text { ative } \\
\text { strains } \\
(4)^{b}\end{array}$ & $\underset{(1)^{b}}{\text { Strain } 33}$ \\
\hline Acid from raffinose & - & + & + \\
\hline $\begin{array}{l}\mathrm{H}_{2} \mathrm{~S} \text { from peptone in } \\
\text { agar }\end{array}$ & + & - & - \\
\hline $\begin{array}{l}\text { Gluconate reduction } \\
\text { (Haynes) }\end{array}$ & + & - & - \\
\hline $\begin{array}{l}\text { Ornithine decarboxyl- } \\
\text { ase }\end{array}$ & $3 / 14+$ & + & + \\
\hline Growth at $44.5 \mathrm{C}$. & $12 / 14+$ & - & - \\
\hline $\begin{array}{l}\text { Growth on } 0.1 \% \text { ce- } \\
\text { trimide }\end{array}$ & + & $1 / 4+$ & + \\
\hline Acid from $5 \%$ sucrose & - & + & + \\
\hline$\beta$-Methyl glucosamine & $12 / 14+$ & - & + \\
\hline
\end{tabular}

${ }^{a}$ The intermediate strain (no. 33) is also included. Key: + , positive; - , negative.

${ }^{b}$ Numbers in parentheses indicate numbers of strains.

pneumoniae, Klebsiella oxytoca, or Klebsiella edwardsii formed a cluster. These strains grouped at $85 \%$ similarity, and, within this cluster, seven strains of $K$. pneumoniae and $K$. oxytoca formed a group at $87 \%$, whereas the two strains of $K$. edwardsii formed a separate group at $86 \%$ before joining the other strains. $K$. oxytoca strains were able to hydrolyze starch, produce indole, and produce acid from $\alpha$-methyl glucosamine but not to produce hydrogen sulfide from cysteine. They were also able to utilize enanthate as a sole source of carbon. The strains received as $K$. pneumoniae did not share these characteristics. On the whole, strains of $K$. pneumoniae and $K$. oxytoca tended to be more biochemically active than those of $K$. edwardsii. $K$. pneumoniae gave positive results for the following characters, whereas negative results were obtained for $K$. edwardsii: acid from lactose and melibiose, growth on $4.5 \%$ sodium chloride, hydrogen sulfide from cysteine, growth on $0.1 \%$ cetrimide and glycerol, and growth on tartrate, benzoate, and hippurate as sole carbon sources.

Preliminary analysis of the data obtained for the 141 strains indicated that the strains received as $C$. freundii and $C$. intermedius should be placed in the same species. $C$. diversus and $L$. malonatica also appeared to be synonymous. $L$. amalonatica, however, was observed to be distinct from this $C$. diversus $/ L$. malonatica group. K. pneumoniae, $K$. oxytoca, and $K$. ed- wardsii appeared to constitute another single species, whereas some strains of $E$. agglomerans shared sufficient characters that they grouped together, although other strains received as $E$. agglomerans or Erwinia species were distinctly different and constituted the majority of the strains which did not belong to any of the 19 clusters.

\section{DISCUSSION}

In general, the results obtained from the phenetic analysis were similar to those obtained previously (38). To determine the stability of the clusters formed by those strains not included in the previous study, all of the strains included in this study were combined with 196 strains whose characteristics have already been reported (38). A computer analysis was undertaken in which the new total of 337 strains (Table 3) was compared over 196 features. Similarities between every pair of strains were calculated using the Jacard coefficient, and subsequent clustering was derived by singlelinkage analysis. A simplified dendrogram showing the arrangement of the strains is illustrated in Fig. 3. The general arrangement of the clusters was observed to be similar to that described previously, as was expected (38).

The main points of interest are as follows: the difference between the two clusters of Citrobacter (C. freundii and Citrobacter spp.); the relationships of the hydrogen sulfide-producing strains of $E$. coli to those strains incapable of producing hydrogen sulfide; the large cluster designated Klebsiella aerogenes and Enterobacter aerogenes; and, lastly, the wide dispersion of strains received as Enterobacter agglomerans or Erwinia species.

All strains received as Citrobacter diversus and Levinea species clustered with the Citrobacter species, although, within this cluster, three strains of $L$. amalonatica again formed a separate subgroup at a very high level of similarity. Strains labeled as $C$. freundii or $C$. intermedius were located in the $C$. freundii cluster.

Strains of Escherichia coli which did not produce hydrogen sulfide (strains $34,35,36,37$, 905-65, 916-65, 897-65, and 193-68) fell in close proximity to the $\mathrm{H}_{2} \mathrm{~S}$-positive strains but were somewhat dispersed. However, they could be distinguished on the basis of selected characters, as shown in Table 2.

The cluster designated Klebsiella aerogenes and Enterobacter aerogenes contained strains 
TABLE 3. Strains assigned to clusters in Fig. 3

\begin{tabular}{|c|c|c|c|}
\hline Cluster identification & $\begin{array}{l}\text { Strain } \\
\text { com- } \\
\text { puter no. }\end{array}$ & Strain received as: & Source $^{2}$ \\
\hline \multirow[t]{6}{*}{ Strains not clustered } & 138 & $\begin{array}{l}\text { Enterobacter agglomerans } 1741- \\
71\end{array}$ & NCDC \\
\hline & 141 & $\begin{array}{l}\text { Enterobacter agglomerans } 1743- \\
71\end{array}$ & NCDC \\
\hline & 2 & Unknown coliform 224 & R. Sakazaki \\
\hline & 1 & Enterobacter liquefaciens 222 & R. Sakazaki \\
\hline & 140 & Erwinia amylovora 1017 & CCM \\
\hline & 139 & Erwinia amylovora 1021 & CCM \\
\hline \multirow[t]{3}{*}{ Proteus rettgeri } & 17 & P. rettgeri 102 & R. Sakazaki \\
\hline & 16 & P. rettgeri 101 & R. Sakazaki \\
\hline & 15 & $P$. rettgeri 103 & R. Sakazaki \\
\hline \multirow[t]{3}{*}{ Proteus mirabilis } & 5 & P. mirabilis 79 & R. Sakazaki \\
\hline & 4 & P. mirabilis 72 & R. Sakazaki \\
\hline & 3 & P. mirabilis 71 & R. Sakazaki \\
\hline \multirow{6}{*}{$\begin{array}{l}\text { Strain not clustered } \\
\text { Yersinia pestis }\end{array}$} & 29 & E. coli $\mathrm{H}_{2} \mathrm{~S}+\mathrm{N} 21$ & R. Sakazaki \\
\hline & & Y. pestis A29 & MRE, Porton \\
\hline & & $Y$. pestis 012 & MRE, Porton \\
\hline & & $Y$. pestis F9581 & MRE, Porton \\
\hline & & Y. pestis EV9-26-70 & NIH, Japan \\
\hline & & Y. pestis A1122 & Fort Collins \\
\hline \multirow{5}{*}{\multicolumn{2}{|c|}{ Yersinia pseudotuberculosis }} & Y. pseudotuberculosis no. 7 & Univ. of Tottori, Japan \\
\hline & & Y. pseudotuberculosis 83 & Univ. of Tottori, Japan \\
\hline & & Y. pseudotuberculosis no. 2 & Univ. of Tottori, Japan \\
\hline & & Y. pseudotuberculosis 1779 & Univ. of Tottori, Japan \\
\hline & & Y. pseudotuberculosis 24 & Univ. of Tottori, Japan \\
\hline \multirow[t]{5}{*}{ Yersinia enterocolitica } & & Y. enterocolitica MYO & Univ. of Lund, Sweden \\
\hline & & Y. enterocolitica MY079 & Univ. of Lund, Sweden \\
\hline & & Y. enterocolitica ALBANY 5189 & Univ. of Lund, Sweden \\
\hline & & Y. enterocolitica LUCAS 110 & Univ. of Lund, Sweden \\
\hline & & Y. enterocolitica 23715 & ATCC \\
\hline \multirow[t]{2}{*}{ Strains not clustered } & 105 & Enterobacter agglomerans 258-71 & NCDC \\
\hline & & Salmonella gallinarum 353 & Costin, Romania \\
\hline \multirow{2}{*}{ Edwardsiella sp. } & & $\begin{array}{l}\text { Edwardsiella sp. } 1-4 \\
\text { Edwardsiella sp } 1-1\end{array}$ & $\begin{array}{l}\text { R. Sakazaki } \\
\text { R. Sakazaki }\end{array}$ \\
\hline & & Edwardsiella sp. 3-1 & R. Sakazaki \\
\hline \multirow{7}{*}{ Edwardsiella tarda } & 11 & E. tarda 1343 & R. Sakazaki \\
\hline & 9 & E. tarda 1223 & R. Sakazaki \\
\hline & 10 & E. tarda 2000 & R. Sakazaki \\
\hline & & E. tarda 41 & R. Sakazaki \\
\hline & & E. tarda 9 & R. Sakazaki \\
\hline & & E. tarda 39 & R. Sakazaki \\
\hline & & E. tarda 51 & R. Sakazaki \\
\hline \multirow[t]{3}{*}{ Proteus morganii } & 6 & P. morganii 2191 & R. Sakazaki \\
\hline & 7 & P. morganii 2192 & R. Sakazaki \\
\hline & 8 & $P$. morganii 2193 & R. Sakazaki \\
\hline \multirow{10}{*}{$\begin{array}{l}\text { Strain not clustered } \\
\text { Shigella spp. }\end{array}$} & 128 & Hafnia alvei 114 & R. Sakazaki \\
\hline & & S. boydii 9770 & NCTC \\
\hline & & S. boydii $2234-60$ & NCDC \\
\hline & & S. dysenteriae 9760 & NCTC \\
\hline & & S. dysenteriae 9720 & NCTC \\
\hline & & S. boydii $1050-50$ & NCDC \\
\hline & & S. boydii $2064-59$ & NCDC \\
\hline & & S. dysenteriae $4379-60$ & NCDC \\
\hline & & S. flexneri 9989 & NCTC \\
\hline & & S. dysenteriae 9719 & NCTC \\
\hline \multirow[t]{3}{*}{ Strains not clustered } & & Shigella flexneri $1 \mathrm{~b}$ 4558-60 & NCDC \\
\hline & & Shigella flexneri $2 \mathrm{~b} 9768$ & NCTC \\
\hline & & Shigella flexneri $2 \mathrm{a}$ 4807-62 & NCDC \\
\hline \multirow[t]{2}{*}{ Shigella sonnei } & & S. sonnei $70-56$ & R. Sakazaki \\
\hline & & S. sonnei $70-69$ & R. Sakazaki \\
\hline
\end{tabular}


TABLE 3-Continued

\begin{tabular}{|c|c|c|c|c|}
\hline Cluster identification & $\begin{array}{c}\text { Strain } \\
\text { com- } \\
\text { puter no. }\end{array}$ & Strain received as: & Source $^{a}$ & \\
\hline & & S. sonnei $70-75$ & R. Sakazaki & \\
\hline & & S. sonnei $70-76$ & R. Sakazaki & \\
\hline \multirow[t]{4}{*}{ Strains not clustered } & & Escherichia coli 905-65 & R. Sakazaki & \\
\hline & & Escherichia coli $916-65$ & R. Sakazaki & \\
\hline & & Escherichia coli 897-65 & R. Sakazaki & \\
\hline & & Escherichia coli $193-68$ & R. Sakazaki & \\
\hline \multirow[t]{15}{*}{ Escherichia coli $\mathrm{H}_{2} \mathrm{~S}+$} & 19 & E. coli $\mathrm{H}_{2} \mathrm{~S}+\mathrm{N} 114$ & R. Sakazaki & \\
\hline & 32 & E. coli $\mathrm{H}_{2} \mathrm{~S}+\mathrm{N} 28$ & R. Sakazaki & \\
\hline & 18 & E. coli $\mathrm{H}_{2} \mathrm{~S}+\mathrm{N} 59$ & R. Sakazaki & \\
\hline & 26 & $E$. coli $\mathrm{H}_{2} \mathrm{~S}+\mathrm{N} 68$ & R. Sakazaki & \\
\hline & 22 & $E$. coli $\mathrm{H}_{2} \mathrm{~S}+\mathrm{N} 46$ & R. Sakazaki & \\
\hline & 31 & $E . \operatorname{coli} \mathrm{H}_{2} \mathrm{~S}+\mathrm{N} 2$ & R. Sakazaki & \\
\hline & 30 & $E$. coli $\mathrm{H}_{2} \mathrm{~S}+\mathrm{N} 5$ & R. Sakazaki & \\
\hline & 28 & E. coli $\mathrm{H}_{2} \mathrm{~S}+\mathrm{N} 63$ & R. Sakazaki & \\
\hline & 24 & E. coli $\mathrm{H}_{2} \mathrm{~S}+\mathrm{N} 73$ & R. Sakazaki & \\
\hline & 23 & E. coli $\mathrm{H}_{2} \mathrm{~S}+\mathrm{N} 76$ & R. Sakazaki & \\
\hline & 27 & E. coli $\mathrm{H}_{2} \mathrm{~S}+\mathrm{N} 99$ & R. Sakazaki & \\
\hline & 25 & E. coli $\mathrm{H}_{2} \mathrm{~S}+\mathrm{N} 103$ & R. Sakazaki & \\
\hline & 29 & $E . \operatorname{coli} \mathrm{H}_{2} \mathrm{~S}+\mathrm{N} 21$ & R. Sakazaki & \\
\hline & 20 & E. coli $\mathrm{H}_{2} \mathrm{~S}+\mathrm{N} 111$ & R. Sakazaki & \\
\hline & 21 & E. coli $\mathrm{H}_{2} \mathrm{~S}+\mathrm{N} 110$ & R. Sakazaki & \\
\hline \multirow[t]{3}{*}{ Salmonella typhi } & 14 & S. typhi 112 & R. Sakazaki & \\
\hline & 13 & S. typhi 111 & R. Sakazaki & \\
\hline & 12 & S. typhi 113 & R. Sakazaki & \\
\hline \multirow[t]{6}{*}{ Strains not clustered } & 35 & E. coli $1324-70$ & R. Sakazaki & \\
\hline & 36 & E. coli $1120-70$ & R. Sakazaki & \\
\hline & 37 & E. coli $1233-70$ & R. Sakazaki & \\
\hline & & S. choleraesuis 1348 & & \\
\hline & 33 & E. coli $1326-70$ & R. Sakazaki & \\
\hline & 34 & E. coli $1441-70$ & R. Sakazaki & \\
\hline \multirow[t]{20}{*}{ Salmonella spp. } & & S. seminore 1685 & $\begin{array}{c}\text { WHO Collabo. } \\
\text { nella Center }\end{array}$ & Salmo- \\
\hline & & S. arizonae 1 & R. Sakazaki & \\
\hline & & S. arizonae 2 & R. Sakazaki & \\
\hline & & S. arizonae 3 & R. Sakazaki & \\
\hline & 57 & S. arizonae 888 & R. Sakazaki & \\
\hline & 58 & Unknown coliform & R. Sakazaki & \\
\hline & 59 & Unknown coliform 114 & R. Sakazaki & \\
\hline & & Unknown coliform 74 & R. Sakazaki & \\
\hline & & Unknown coliform 75 & R. Sakazaki & \\
\hline & & S. chameleon & $\begin{array}{c}\text { WHO Collabo. } \\
\text { nella Center }\end{array}$ & Salmo- \\
\hline & & S. ochsenzoll & $\begin{array}{c}\text { WHO Collabo. } \\
\text { nella Center }\end{array}$ & Salmo- \\
\hline & & S. soesterberg & $\begin{array}{c}\text { wHO Collabo. } \\
\text { nella Center }\end{array}$ & Salmo- \\
\hline & & S. parera & $\begin{array}{c}\text { WHO Collabo. } \\
\text { nella Center }\end{array}$ & Salmo- \\
\hline & & S. bonaire & $\begin{array}{c}\text { WHO Collabo. } \\
\text { nella Center }\end{array}$ & Salmo- \\
\hline & & S. houten & WHO Collabo. & Salmo- \\
\hline & & & nella Center & \\
\hline & & S. mundsberg & $\begin{array}{c}\text { WHO Collabo. } \\
\text { nella Center }\end{array}$ & Salmo- \\
\hline & & S. tuindorp & $\begin{array}{l}\text { WHO Collabo. } \\
\text { nella Center }\end{array}$ & Salmo- \\
\hline & & S. argentina & WHO Collabo. & Salmo- \\
\hline & & S. beloha & $\begin{array}{c}\text { nella Center } \\
\text { WHO Collabo. } \\
\text { nella Center }\end{array}$ & Salmo- \\
\hline
\end{tabular}


TABle 3-Continued

\begin{tabular}{|c|c|c|c|c|}
\hline Cluster identification & $\begin{array}{l}\text { Strain } \\
\text { com- }\end{array}$ & Strain received as: & Source $^{a}$ & \\
\hline & & S. eilbek & $\begin{array}{c}\text { WHO Collabo. } \\
\text { nella Center }\end{array}$ & Salmo- \\
\hline . & & S. nairobi & $\begin{array}{c}\text { WHO Collabo. } \\
\text { nella Center }\end{array}$ & Salmo- \\
\hline & & S. bilthoven & $\begin{array}{c}\text { WHO Collabo. } \\
\text { nella Center }\end{array}$ & Salmo- \\
\hline & & S. ngozi & $\begin{array}{c}\text { WHO Collabo. } \\
\text { nella Center }\end{array}$ & Salmo- \\
\hline & & S. basel & $\begin{array}{c}\text { WHO Collabo. } \\
\text { nella Center }\end{array}$ & Salmo- \\
\hline & & S. artis & $\begin{array}{c}\text { WHO Collabo. } \\
\text { nella Center }\end{array}$ & Salmo- \\
\hline & & S. locarno & $\begin{array}{c}\text { WHO Collabo. } \\
\text { nella Center }\end{array}$ & Salmo- \\
\hline & & S. setubal & $\begin{array}{c}\text { WHO Collabo. } \\
\text { nella Center }\end{array}$ & Salmo- \\
\hline & & S. haarlem & $\begin{array}{c}\text { WHO Collabo. } \\
\text { nella Center }\end{array}$ & Salmo- \\
\hline & 55 & S. infantis & R. Sakazaki & \\
\hline & 54 & S. derby & R. Sakazaki & \\
\hline & & S. krefeld & R. Sakazaki & \\
\hline & & S. kiambu & R. Sakazaki & \\
\hline & & S. meieagridis & R. Sakazaki & \\
\hline & & S. montivideo & R. Sakazaki & \\
\hline & & S. typhimurium & R. Sakazaki & \\
\hline & 53 & S. meleagridis & R. Sakazaki & \\
\hline & & S. enteritidis & R. Sakazaki & \\
\hline & & S. anatum & R. Sakazaki & \\
\hline & & S. livingstone & R. Sakazaki & \\
\hline & & S. derby & R. Sakazaki & \\
\hline & & S. infantis. & R. Sakazaki & \\
\hline & & S. bredeney & R. Sakazaki & \\
\hline & & S. heidelberg & R. Sakazaki & \\
\hline & 52 & S. enteritidis & R. Sakazaki & \\
\hline & 56 & S. livingstone & R. Sakazaki & \\
\hline Strain not clustered & & Citrobacter freundii 10 & R. Sakazaki & \\
\hline Citrobacter freundii & 50 & C. intermedius 3 & R. Sakazaki & \\
\hline & & C. freundii 1 & R. Sakazaki & \\
\hline & 45 & C. intermedius 5 & R. Sakazaki & \\
\hline & 43 & C. freundii $423-71$ & R. Sakazaki & \\
\hline & & C. freundii 6 & R. Sakazaki & \\
\hline & 49 & C. freundii $624-71$ & R. Sakazaki & \\
\hline & 44 & C. freundii $511-71$ & R. Sakazaki & \\
\hline & & C. freundii 5 & R. Sakazaki & \\
\hline & & C. freundii 3 & R. Sakazaki & \\
\hline & & C. freundii 2 & R. Sakazaki & \\
\hline & & C. freundii 4 & R. Sakazaki & \\
\hline & & C. freundii 9 & R. Sakazaki & \\
\hline & & C. freundii 12 & R. Sakazaki & \\
\hline & & C. freundii 7 & R. Sakazaki & \\
\hline & 42 & C. freundii $577-67$ & R. Sakazaki & \\
\hline & 47 & C. intermedius 4 & R. Sakazaki & \\
\hline & 46 & C. intermedius 1 & R. Sakazaki & \\
\hline Strains not clustered & 48 & C. intermedius 2 & R. Sakazaki & \\
\hline & 51 & C. freundii $624-70$ & R. Sakazaki & \\
\hline & 93 & Erwinia carotovora 979 & CCM & \\
\hline Citrobacter spp. & 76 & C. diversus $2469-70$ & NCDC & \\
\hline & 64 & Levinea malonatica $527-71$ & R. Sakazaki & \\
\hline & 63 & Levinea malonatica 25409 & ATCC & \\
\hline
\end{tabular}


TABLE 3-Continued

\begin{tabular}{|c|c|c|c|}
\hline Cluster identification & $\begin{array}{c}\text { Strain } \\
\text { com- } \\
\text { puter no. }\end{array}$ & Strain received as: & Source $^{a}$ \\
\hline & 74 & Levinea malonatica $528-71$ & R. Sakazaki \\
\hline & 72 & Levinea malonatica 25408 & ATCC \\
\hline & 73 & Levenia malonatica 25410 & ATCC \\
\hline & 65 & C. diversus $1066-70$ & NCDC \\
\hline & 68 & C. diversus $3613-63$ & NCDC \\
\hline \multirow[t]{10}{*}{ Citrobacter diversus } & 69 & C. diversus $2292-70$ & NCDC \\
\hline & 67 & C. diversus $2737-70$ & NCDC \\
\hline & 71 & C, diversus $2524-70$ & NCDC \\
\hline & 66 & C. diversus $2572-70$ & NCDC \\
\hline & 75 & C. diversus $1381-70$ & $\mathrm{NCDC}$ \\
\hline & 70 & C. diversus $2393-70$ & NCDC \\
\hline & 77 & C. diversus $2555-70$ & NCDC \\
\hline & 62 & Levinea amalonatica 25407 & ATCC \\
\hline & 61 & Levinea amalonatica 25405 & ATCC \\
\hline & 60 & Levinea amalonatica 25406 & ATCC \\
\hline \multirow{4}{*}{ Salmonella spp. } & & S. sendai $618-65$ & R. Sakazaki \\
\hline & & S. pullorum 1334-D67 & Costin, Romania \\
\hline & & S. paratyphi A T67-131 & R. Sakazaki \\
\hline & & S. abortusequi WH2 & R. Sakazaki \\
\hline \multirow[t]{16}{*}{ Hafnia alvei } & & H. alvei 56211 & Eveland \\
\hline & & H. alvei P344 & Eveland \\
\hline & & H. alvei $100-794$ & Eveland \\
\hline & & H. alvei $887-61$ & R. Sakazaki \\
\hline & & H. alvei 70811 & Eveland \\
\hline & 38 & H. alvei 13 & R. Sakazaki \\
\hline & 39 & H. alvei 7081 & R. Sakazaki \\
\hline & 40 & $H$. alvei 5621 & R. Sakazaki \\
\hline & & H. alvei $441-61$ & R. Sakazaki \\
\hline & 41 & H. alvei $887-61$ & R. Sakazaki \\
\hline & & H. alvei $1190-61$ & R. Sakazaki \\
\hline & & H. alvei $732-61$ & R. Sakazaki \\
\hline & & H. alvei P253 & Eveland \\
\hline & & H. alvei $\mathrm{P} 228$ & Eveland \\
\hline & & Enterobacter cloacae 2-55 & R. Sakazaki \\
\hline & 79 & Enterobacter cloacae 116 & R. Sakazaki \\
\hline \multirow[t]{13}{*}{ Enterobacter cloacae } & 80 & E. cloacae 101-71 & R. Sakazaki \\
\hline & & E. cloacae $717-53$ & R. Sakazaki \\
\hline & 81 & E. cloacae 1679 & R. Sakazaki \\
\hline & 82 & E. cloacae 2011 & R. Sakazaki \\
\hline & 78 & E. cloacae 102-71 & R. Sakazaki \\
\hline & & E. cloacae SA 153 & R. Sakazaki \\
\hline & & E. cloacae $558-53$ & R. Sakazaki \\
\hline & & E. cloacae 116-54 & R. Sakazaki \\
\hline & & E. cloacae $0427-53$ & R. Sakazaki \\
\hline & & E. cloacae $1366-53$ & R. Sakazaki \\
\hline & & E. cloacae $1609-53$ & R. Sakazaki \\
\hline & & E. cloacae $1302-54$ & R. Sakazaki \\
\hline & & E. cloacae 2015-53 & R. Sakazaki \\
\hline \multirow{11}{*}{$\begin{array}{l}\text { Klebsiella and Enterobacter } \\
\text { aerogenes }\end{array}$} & & $E$. aerogenes $114-54$ & R. Sakazaki \\
\hline & & E. aerogenes $2-56$ & R. Sakazaki \\
\hline & & E. aerogenes 1414-69 & R. Sakazaki \\
\hline & & $E$. aerogenes $1412-69$ & R. Sakazaki \\
\hline & & E. aerogenes 138-54 & R. Sakazaki \\
\hline & & E. aerogenes 195-57 & R. Sakazaki \\
\hline & & E. aerogenes $1405-69$ & R. Sakazaki \\
\hline & 100 & K. edwardsii 9496 & NCTC \\
\hline & 96 & K. oxytoca 13183 & ATCC \\
\hline & 98 & K. oxytoca 13182 & ATCC \\
\hline & 97 & K. oxytoca $833-64$ & R. Sakazaki \\
\hline
\end{tabular}


TABLE 3-Continued

\begin{tabular}{|c|c|c|c|}
\hline Cluster identification & $\begin{array}{c}\text { Strain } \\
\text { com- } \\
\text { puter no. }\end{array}$ & Strain received as: & Source $^{a}$ \\
\hline Klebsiella ozaenae & $\begin{array}{r}127 \\
83 \\
84 \\
102 \\
125 \\
123 \\
122 \\
121 \\
124\end{array}$ & 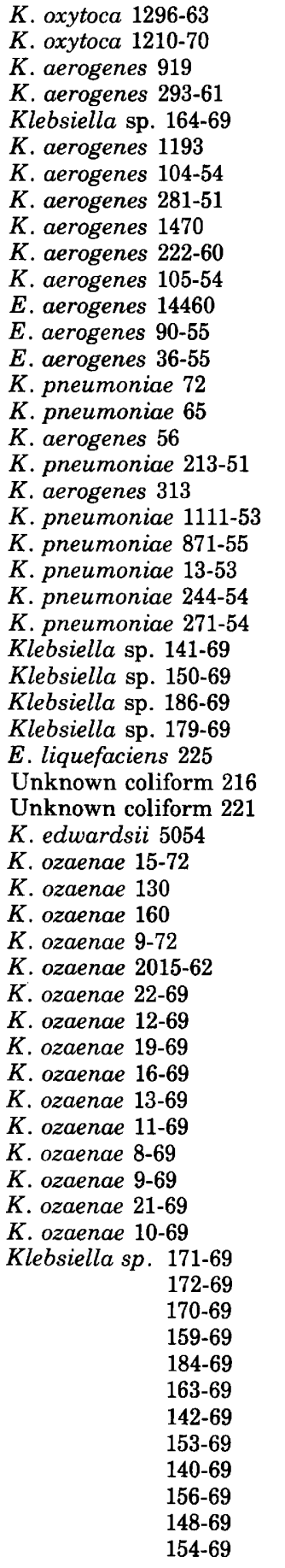 & $\begin{array}{l}\text { R. Sakazaki } \\
\text { R. Sakazaki } \\
\text { R. Sakazaki } \\
\text { R. Sakazaki } \\
\text { R. Sakazaki } \\
\text { R. Sakazaki } \\
\text { R. Sakazaki } \\
\text { R. Sakazaki } \\
\text { R. Sakazaki } \\
\text { R. Sakazaki } \\
\text { R. Sakazaki } \\
\text { ATCC } \\
\text { R. Sakazaki } \\
\text { R. Sakazaki } \\
\text { R. Sakazaki } \\
\text { R. Sakazaki } \\
\text { R. Sakazaki } \\
\text { R. Sakazaki } \\
\text { R. Sakazaki } \\
\text { R. Sakazaki } \\
\text { R. Sakazaki } \\
\text { R. Sakazaki } \\
\text { R. Sakazaki } \\
\text { R. Sakazaki } \\
\text { R. Sakazaki } \\
\text { R. Sakazaki } \\
\text { R. Sakazaki } \\
\text { R. Sakazaki } \\
\text { R. Sakazaki } \\
\text { R. Sakazaki } \\
\text { R. Sakazaki } \\
\text { NCTC } \\
\text { R. Sakazaki } \\
\text { R. Sakazaki } \\
\text { R. Sakazaki } \\
\text { R. Sakazaki } \\
\text { R. Sakazaki } \\
\text { R. Sakazaki } \\
\text { R. Sakazaki } \\
\text { R. Sakazaki } \\
\text { R. Sakazaki } \\
\text { R. Sakazaki } \\
\text { R. Sakazaki } \\
\text { R. Sakazaki } \\
\text { R. Sakazaki } \\
\text { R. Sakazaki } \\
\text { R. Sakazaki } \\
\text { R. Sakazaki } \\
\text { R. Sakazaki } \\
\text { R. Sakazaki } \\
\text { R. Sakazaki } \\
\text { R. Sakazaki } \\
\text { R. Sakazaki } \\
\text { R. Sakazaki } \\
\text { R. Sakazaki } \\
\text { R. Sakazaki } \\
\text { R. Sakazaki } \\
\text { R. Sakazaki } \\
\text { R. Sakazaki }\end{array}$ \\
\hline
\end{tabular}


TABLE 3-Continued

\begin{tabular}{|c|c|c|c|}
\hline Cluster identification & $\begin{array}{c}\begin{array}{c}\text { Strain } \\
\text { com- } \\
\text { puter no. }\end{array} \\
\end{array}$ & Strain received as: & Source $^{a}$ \\
\hline & & $144-69$ & R. Sakazaki \\
\hline & & $133-69$ & R. Sakazaki \\
\hline & & $177-69$ & R. Sakazaki \\
\hline \multirow{14}{*}{ Klebsiella rhinoscleromatis } & 119 & K. rhinoscleromatis i-234-53 & Orskov \\
\hline & 112 & $K$. rhinoscleromatis i-1-50 & Orskov \\
\hline & 111 & $K$. rhinoscleromatis i-501-56 & Drskov \\
\hline & 116 & $K$. rhinoscleromatis i-9-50 & Orskov \\
\hline & 115 & $K$. rhinoscleromatis i-6-50 & Orskov \\
\hline & 114 & K. rhinoscleromatis i-255-53 & Orskov \\
\hline & 113 & K. rhinoscleromatis i-236-53 & Orskov \\
\hline & 118 & K. rhinoscleromatis i-256-53 & Qrskov \\
\hline & 117 & K. rhinoscleromatis i-235-53 & Qrskov \\
\hline & 120 & K. rhinoscleromatis i $11-50$ & Ørskov \\
\hline & & K. rhinoscleromatis $1218-53$ & R. Sakazaki \\
\hline & & $K$. rhinoscleromatis $136-55$ & R. Sakazaki \\
\hline & & K. rhinoscleromatis $119-53$ & R. Sakazaki \\
\hline & & K. rhinoscleromatis $1230-54$ & R. Sakazaki \\
\hline \multirow[t]{12}{*}{ Enterobacter liquefaciens } & 130 & E. liquefaciens 1689 & R. Sakazaki \\
\hline & 129 & E. liquefaciens 2214 & R. Sakazaki \\
\hline & 131 & E. liquefaciens 1493 & R. Sakazaki \\
\hline & & E. liquefaciens 1111-65 & R. Sakazaki \\
\hline & & E. liquefaciens $1689-69$ & R. Sakazaki \\
\hline & & E. liquefaciens $1638-69$ & R. Sakazaki \\
\hline & & E. liquefaciens $2214-69$ & R. Sakazaki \\
\hline & & E. liquefaciens $2108-69$ & R. Sakazaki \\
\hline & & E. liquefaciens $1493-69$ & R. Sakazaki \\
\hline & & E. liquefaciens $2318-68$ & R. Sakazaki \\
\hline & & E. liquefaciens $1312-67$ & R. Sakazaki \\
\hline & & E. liquefaciens $1783-67$ & R. Sakazaki \\
\hline \multirow[t]{5}{*}{ Strains not clustered } & 103 & Unknown coliform 222 & R. Sakazaki \\
\hline & 85 & Unknown coliform 215 & R. Sakazaki \\
\hline & 92 & Unknown coliform 213 & R. Sakazaki \\
\hline & & Enterobacter liquefaciens D974 & R. Sakazaki \\
\hline & 90 & Enterobacter agglomerans 459-71 & NCDC \\
\hline \multirow[t]{15}{*}{ Serratia marcescens } & & S. marcescens $3130-68$ & R. Sakazaki \\
\hline & & S. marcescens $2187-68$ & R. Sakazaki \\
\hline & & S. marcescens $2249-68$ & R. Sakazaki \\
\hline & & S. marcescens $2630-68$ & R. Sakazaki \\
\hline & & S. marcescens $2191-68$ & R. Sakazaki \\
\hline & & S. marcescens $2241-68$ & R. Sakazaki \\
\hline & & S. marcescens $2638-68$ & R. Sakazaki \\
\hline & & S. marcescens $2242-68$ & R. Sakazaki \\
\hline & & S. marcescens $2963-68$ & R. Sakazaki \\
\hline & & S. marcescens 2121-68 & R. Sakazaki \\
\hline & 135 & S. marcescens $2249-68$ & R. Sakazaki \\
\hline & 136 & S. marcescens 14 & R. Sakazaki \\
\hline & 134 & S. marcescens $2187-68$ & R. Sakazaki \\
\hline & 133 & S. marcescens 16 & R. Sakazaki \\
\hline & 132 & S. marcescens $2121-68$ & R. Sakazaki \\
\hline \multirow[t]{9}{*}{ Strains not clustered } & 91 & Enterobacter agglomerans 219-71 & NCDC \\
\hline & 89 & Enterobacter agglomerans $185-71$ & NCDC \\
\hline & 88 & $\begin{array}{l}\text { Enterobacter agglomerans } 1379- \\
71\end{array}$ & NCDC \\
\hline & 86 & Enterobacter agglomerans 184-71 & NCDC \\
\hline & 126 & Erwinia herbicola Aj 2671 & Tokyo Univ. \\
\hline & 108 & Erwinia herbicola Aj 2189 & Tokyo Univ. \\
\hline & 109 & Erwinia herbicola Aj 2190 & Tokyo Univ. \\
\hline & 87 & Enterobacter agglomerans 6-71 & NCDC \\
\hline & 110 & Enterobacter agglomerans 5379- & NCDC \\
\hline
\end{tabular}


TABLE $3-$ Continued

\begin{tabular}{llll}
\hline Cluster identification & $\begin{array}{c}\text { Strain } \\
\text { com- } \\
\text { puter no. }\end{array}$ & Strain received as: & Source $^{a}$ \\
& 107 & $\begin{array}{c}\text { Erwinia carotovora } 1012 \\
\text { Erwinia carotovora 1016 }\end{array}$ & CCM \\
& 106 & CCM \\
104 & $\begin{array}{l}\text { Erwinia carotovora } 630 \\
\text { Enterobacter agglomerans } 3737-\end{array}$ & NCDC \\
& 137 & 71 & \\
\hline
\end{tabular}

${ }^{a}$ Abbreviations: Ørskov, F. Orskov, WHO International Escherichia Reference Center, Statens Seruminstitut, Amager Bld. 80, 2300 Copenhagen 5, Denmark; NIH, Tokyo, National Institute of Health of Japan, 284, Kamiosaki-Chojamaru, Shinagawa-Ku, Tokyo; Eveland, W. C. Eveland, 406th Medical General Laboratory, U. S. Army, A.P.O. 500, c/o Postmaster, San Francisco, Calif.; Costin, Romania, I. D. Costin, The State Inspection for Hygiene, Timisoara, Romania; WHO Collabo. Salmonella Center, World Health Organization Collaborative Salmonella Center, Institut Pasteur, Paris; NCDC, National Center for Disease Control, Atlanta, Ga.; NCTC, National Collection of Type Cultures, Central Public Health Laboratory, Colindale Avenue, London NW 9, England; Univ. of Tottori, University of Tottori, 1-6-chome, Tachikawacho, Tottori-shi, Japan; M.R.E. Porton, Microbiological Research Establishment, Porton Down, Wiltshire, England; Fort Collins, Colorado State University, Fort Collins, Colo.; Univ. of Lund, Lund Universitet, Fack, 22101 Lund 1, Sweden; ATCC, American Type Culture Collection, 12301 Parklawn Drive, Rockville, Md.; CCM, Czechoslovak Collection of Miroorganisms, J. E. Purkyne University, tř, Obráncu míru 10, Brno, Czechoslovakia; Tokyo Univ., University of Tokyo, 7-3-1 Hongo, Bunkyo-ku, Tokyo 113, Japan.

received as $K$. oxytoca, $K$. edwardsii, $K$. pneumoniae, $K$. aerogenes, and $E$. aerogenes. This arrangement was consistent in the earlier study as well as in this analysis.

Classification of the genus Citrobacter. The history of the genus Citrobacter centers around one well-established species, $C$. freundii (Braak) Werkman and Gillen 1932, and involves the search for a suitable species to accommodate those strains which do not produce hydrogen sulfide. The nomenclature of the group has been reviewed by Frederiksen (28) and by Ewing and Davis (22), although differing conclusions were reached. Recently, Sedlák (58) reintroduced $C$. intermedius in the 8th edition of Bergey's Manual of Determinative Bacteriology, making an already confusing situation more difficult. Results of important contributions to the taxonomy of Citrobacter by various investigators are summarized in Table 4.

Werkman and Gillen (67) proposed the genus Citrobacter based on studies of 15 strains. They subdivided the genus into seven species: $C$. freundii, C. album, C. glycologenes, C. intermedium, $C$. decolorans, $C$. diversum, and $C$. anindolicum. However, only three species have been widely accepted.

Werkman and Gillen's description of $C$. freundii was based on two strains. Nevertheless, it has now become recognized as a legitimate species, although description of the species has been broadened on the basis of studies on larger numbers of strains (20). Their description of Citrobacter intermedium was based on four strains, and the species description differed from $C$. freundii in several characteristics (see Table 5). Tittsler and Sandholzer (64) examined two of the strains (M8BK and 24R) which Werkman and Gillen assigned to $C$. intermedium and noted that both produced acid from salicin and hydrogen sulfide on lead acetate agar (the medium used by Werkman and Gillen).

It has since been confirmed by other authors that strain M8BK does, in fact, produce hydrogen sulfide and is capable of acidifying salicin. Detailed characterization of this strain at the American Type Culture Collection (ATCC 6750) indicated that it is a typical strain of $C$. freundii, as this species is presently defined (15). Table 5 shows characteristics employed by Werkman and Gillen for differentiation of $C$. freundii from C. intermedium. Also included in the table are results for the same characteristics given for $C$. freundii, as defined by Ewing. The single difference between $C$. intermedium and Ewing's definition of $C$. freundii is the production of hydrogen sulfide, the characteristic that Werkman and Gillen had recorded incorrectly. The differences between $C$. freundii of Werkman and Gillen and $C$. intermedium were more apparent than real, and they recede when larger numbers of strains are examined. In addition, the only authentic strain of $C$. intermedium now extant (ATCC 6750) has been shown to be a typical $C$. freundii. Thus, we must conclude that the two species are synonymous names, and we propose that $C$. freundii be retained on the grounds of priority.

Macierewicz (50) studied 27 strains which 


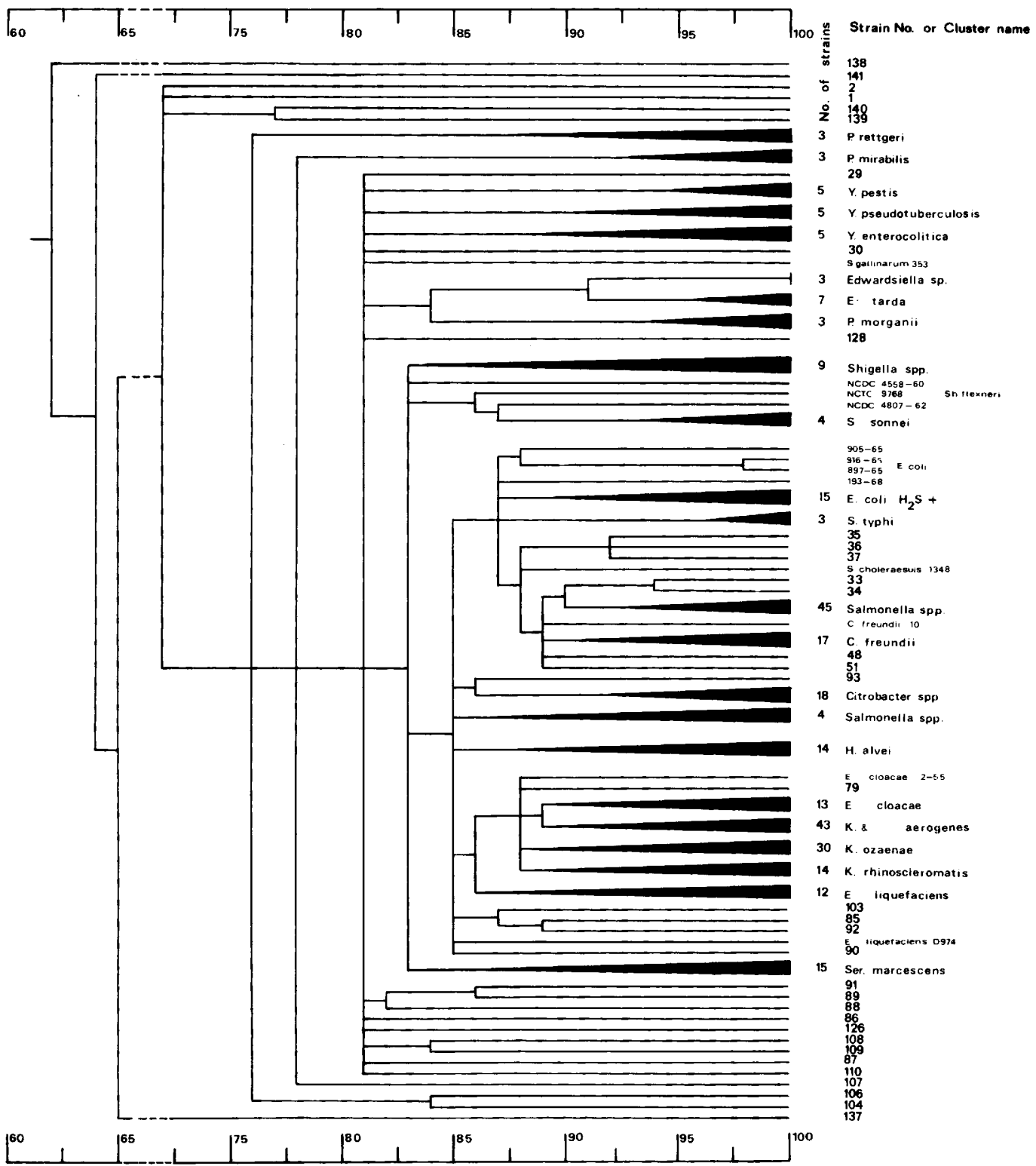

FIG. 3. Taxonomic relationships among species of Enterobacteriaceae, Yersinia, and related organisms included in a combined analysis of a total of 337 strains.

failed to produce hydrogen sulfide. These were assigned to a new genus, Padlewskia. However, Macierewicz failed to propose any species for this genus. Frederiksen (28) proposed a new species, Citrobacter koseri, for 30 strains similar to those of Macierewicz, although they differed in the production of acid from adonitol, gelatin hydrolysis, and growth in Møller po- tassium cyanide medium (see Table 4). In a study of 108 strains, Young et al. (68) proposed a new genus, Levinea, with two species, $L$. $m a$ lonatica and L. amalonatica. Examination of Table 4 indicates that $L$. malonatica is identical to $C$. koseri, whereas $L$. amalonatica is identical to Padlewskia, a conclusion shared by Sedlák (58). C. koseri has priority over L. ma- 


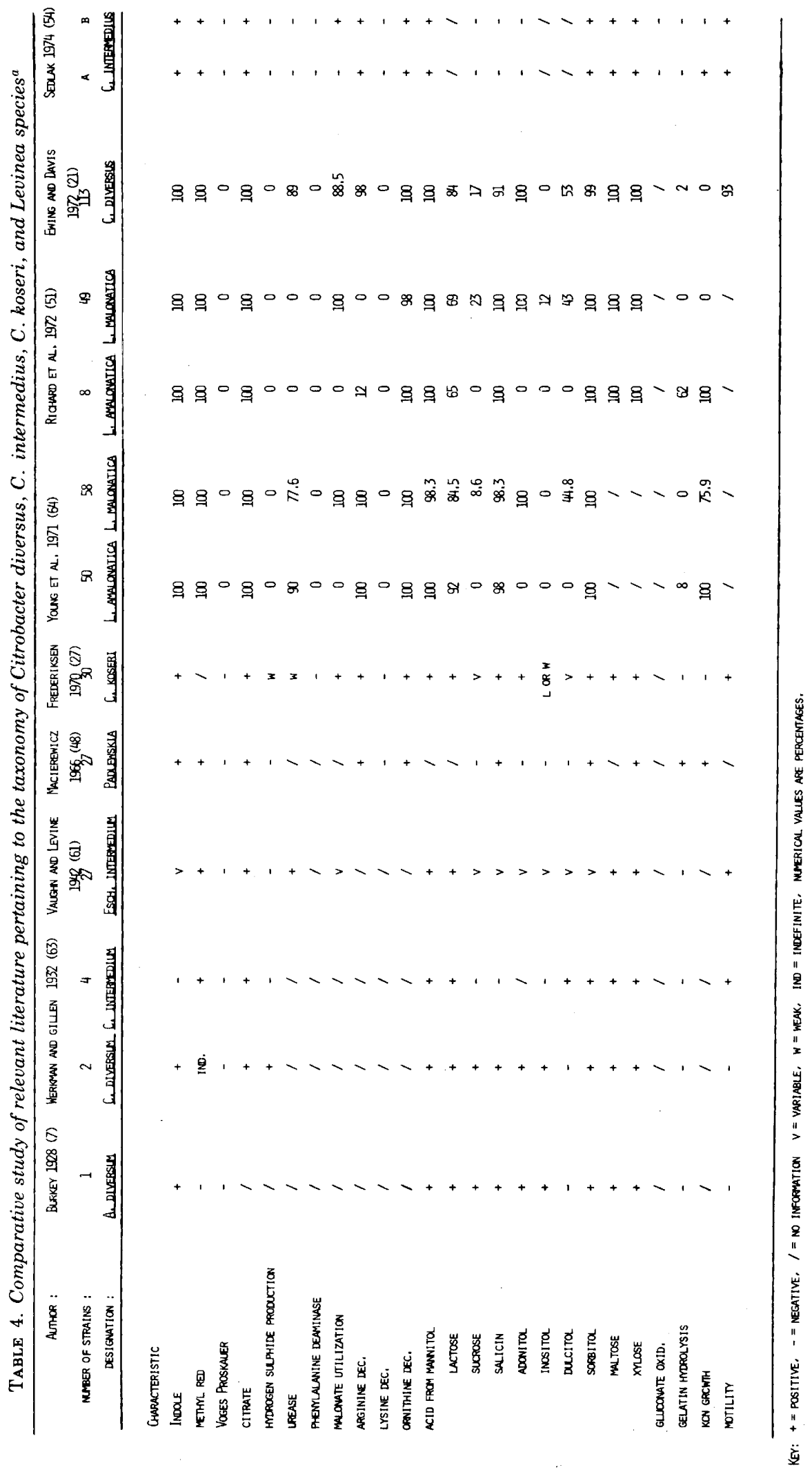


TABLE 5. Comparison of three species of Citrobacter, as described by Werkman and Gillen, with the evolved description of $C$. freundii ${ }^{a}$

\begin{tabular}{lcccc}
\hline & $\begin{array}{c}\text { Ewing } \\
\text { Character }\end{array}$ & \multicolumn{3}{c}{ Werkman and Gillen 1932 (67) } \\
\cline { 3 - 5 } & $\begin{array}{c}\text { C. freun- } \\
\text { dii }\end{array}$ & $\begin{array}{c}\text { C. freun- } \\
\text { dii }\end{array}$ & $\begin{array}{c}\text { C. inter } \\
\text { medium }\end{array}$ & $\begin{array}{c}\text { C. diver- } \\
\text { sum }\end{array}$ \\
\hline Indole & - & Weak & - & + \\
Methyl red & + & + & + & Indefinite \\
H$_{2}$ S & $(+)$ & + & - & + \\
Motility & + & + & + & - \\
Esculin & - & + & - & + \\
Sucrose & $\mathrm{V}$ & + & - & + \\
Salicin & $\mathrm{V}$ & + & - & + \\
Galactose & I & + & + & - \\
Dulcitol & $\mathrm{V}$ & - & + & - \\
Inositol & - & + & - & + \\
Raffinose & $\mathrm{V}$ & + & - & + \\
Glycogen & I & - & $/$ & + \\
Melezitose & I & - & - & + \\
\hline
\end{tabular}

${ }^{a}$ Key: +, positive; -, negative; /, no information; $\mathrm{V}$, variable; $(+)$, delayed positive.

lonatica, whereas L. amalonatica should be retained over Padlewskia, since a type species of the latter was not proposed.

Ewing and Davis (22) also reported on strains similar to those described by Young et al. (68) and Frederiksen (28). Examination of the literature led them to conclude that the name $C$. diversus (Burkey) Werkman and Gillen was the appropriate species for many of the isolates, although they commented that there were some differences between the description provided by Werkman and Gillen and their own. Table 4 shows that $C$. diversus, as described by Ewing and Davis, is significantly similar in its characteristics to C. koseri and L. malonatica. Therefore, the logical conclusion is to consider these synonymous. It can be argued that the differences between the description by Werkman and Gillen and that by Ewing and Davis are sufficiently great to preclude consideration of the strains as belonging to the same species. Werkman and Gillen, for example, recorded $C$. diversum as nonmotile, giving an indefinite methyl red reaction, producing hydrogen sulfide, and not producing acid from inositol. The difference in hydrogen sulfide production can be explained by the test methods employed, since the lead acetate agar used by Werkman and Gillen is more sensitive than the triple sugar iron medium used by Ewing and Davis. On the basis of data for the two strains included in this study, we do not feel that the other characters are sufficient in number to determine that the strains are different species. Comparison of $C$. diversus, $L$. malonatica, and C. koseri gives a strong indication that all of those strains belong to the same species and that $C$. diversus has priority. The argument that the original description of $C$. diversum may be inadequate appears dubious, even though some characteristics such as amino acid decarboxylase reactions were not recorded.

Ewing and Davis (22) also reported that strains of L. amalonatica and Padlewskia appeared to be identical to strains of $C$. freundii that were hydrogen sulfide negative and indole positive. Even though they retained these strains as variants of $C$. freundii, evidence appears to be accumulating that suggests either that there is a closer relationship to $C$. diversus or that these strains should be considered a separate species, intermediate between $C$. freundii and $C$. diversus. The phenetic analysis used in this study resulted in separation of $L$. amalonatica from the $C$. diversus group. Gross and Rowe (33) reported that strains of $L$. amalonatica possessed common $\mathrm{O}$ antigens not shared with $L$. malonatica, C. koseri, or C. diversus, although strains received under the latter names did share common $\mathrm{O}$ antigens. Perhaps the strongest evidence is provided by Crosa et al. (13) in deoxyribonucleic acid (DNA)-DNA homology studies of these organisms. Table 6 summarizes the ranges of homologies obtained at the less stringent temperature, i.e., $60 \mathrm{C}$. The results clearly show a division of the genus Citrobacter into three groups: $C$. freundii and some biochemically and serologically atypical strains; $L$. amalonatica; and $C$. diversus. The degree of relatedness appears to be similar among all of the groups. Results of this study are in agreement with those of Crosa et al., namely that the genus Citrobacter should consist of three taxa: $C$. freundii, $C$. diversus, and $L$. amalonatica. The authors postpone the naming of $L$. amalonatica as a species of Citrobacter until the legitimacy of the specific epithet "amalonatica" has been studied.

Hydrogen sulfide variants of Escherichia coli. Judging by overall phenetic similarity and the individual biochemical test results, there is little doubt but that the hydrogen sulfide-producing strains of Escherichia coli belong with the more typical strains within the same species. The possibility exists, however, that they may be confused with Citrobacter. Some of the more useful differentiating characteristics are shown in Table 7. Lautrop et al. (46) and Darland and Davis (14) reported the occurrence of $\mathrm{H}_{2} \mathrm{~S}$-positive strains. Thus, it appears that these strains represent a very small proportion of $E$. coli strains isolated from clinical specimens. They also exhibit biochemical and serological variability and do not represent a restricted biotype of the species. The property may sometimes be lost and can also be transferred to $\mathrm{H}_{2} \mathrm{~S}$-negative strains; it has been 
TABLE 6. DNA-DNA homologies determined among Citrobacter and Levinea spp. at $60 \mathrm{C}^{a}$

\begin{tabular}{lcccc}
\hline \multirow{2}{*}{ Species } & \multicolumn{4}{c}{ DNA homology } \\
\cline { 2 - 5 } & C. freundii & $\begin{array}{c}\text { C. freundii } \\
\text { (atypical) }\end{array}$ & L. amalonatica & L. malonatica \\
\hline C. freundii & $90-100$ & $69-77$ & $53-56$ & $51-58$ \\
C. freundii (atypical) & $76-97$ & $68-100$ & $\mathbf{5 3 - 5 5}$ & $\mathbf{5 2 - 5 8}$ \\
L. amalonatica & $62-66$ & $56-57$ & $94-100$ & $56-66$ \\
L. malonatica & $63-68$ & $\mathbf{5 5 - 5 9}$ & $\mathbf{5 7 - 6 3}$ & $86-100$ \\
C. diversus & $65-70$ & $59-61$ & $\mathbf{5 7 - 5 8}$ & $\mathbf{8 3 - 8 5}$ \\
\hline
\end{tabular}

a Data summarized from Crosa et al. (13).

TABLE 7. Selected characteristics differentiating hydrogen sulfide variants of Escherichia coli from citrobacters ${ }^{a}$

\begin{tabular}{|c|c|c|c|c|c|}
\hline \multirow[b]{2}{*}{ Character } & \multicolumn{2}{|c|}{ E. coli } & \multirow[b]{2}{*}{ C. freundii } & \multirow[b]{2}{*}{ C. diversus } & \multirow[b]{2}{*}{ C. amalonatica } \\
\hline & $\begin{array}{c}\mathrm{H}_{2} \mathrm{~S} \\
\text { positive }\end{array}$ & $\begin{array}{c}\mathrm{H}_{2} \mathrm{~S} \\
\text { negative }\end{array}$ & & & \\
\hline Indole & + & + & - & + & + \\
\hline Methyl red & + & + & + & + & + \\
\hline Voges-Proskauer & - & - & - & - & - \\
\hline Lysine dec. & + & + & - & - & - \\
\hline Ornithine dec. & $5 / 14$ & + & - & + & + \\
\hline $\mathrm{KCN}$ & - & - & + & - & + \\
\hline Malonate & & & $\mathrm{V}$ & + & - \\
\hline $\mathrm{H}_{2} \mathrm{~S}$ from thiosulfate & + & - & + & - & - \\
\hline Gluconate & + & - & $7 / 17$ & - & - \\
\hline Starch hydrolysis & + & - & - & - & - \\
\hline Acid from raffinose & - & + & - & - & - \\
\hline Acid from $5 \%$ sucrose & + & - & 1 & 1 & 1 \\
\hline Acid from adonitol & - & - & - & - & + \\
\hline
\end{tabular}

${ }^{a}$ For key to symbols, see footnote $a$, Table 5 .

shown that the ability to produce hydrogen sulfide is associated with a plasmid (54).

The enigma of the clustering of Klebsiella aerogenes and Enterobacter aerogenes remains to be solved. In our previous study, we argued in favor of retaining $K$. pneumoniae for a group of strains received as $K$. pneumoniae, $K$. aerogenes, and some unidentified Klebsiella species (38). In this study, a similar cluster was formed, but in addition to the above-named strains, $K$. oxytoca and $K$. edwardsii also fell into this group. Kaluzewski (39) favored granting specific status to $K$. oxytoca for the indolepositive strains. Ørskov (53) retained the name $K$. oxytoca for those strains which are indole positive and liquefy gelatin. On the other hand, Cowan et al. (12) followed the earlier dictum of Lautrop (45) and excluded $K$. oxytoca from the genus altogether. They subdivided 176 strains of Klebsiella into six groups, differentiated on the basis of 13 characteristics. Four groups were assigned specific rank: $K$. aerogenes, $K$. pneumoniae (sensu stricto), $K$. rhinoscleromatis, and $K$. ozaenae, whereas the other two groups were given varietal status in a new species, $K$. edwardsii. The results of a phenetic analysis
(3) showed unequivocally that strains of $K$. aerogenes, $K$. pneumoniae (sensu stricto), $K$. oxytoca, and $K$. edwardsii were highly related, with similarities of $>90 \%$. It was conservatively argued that $K$. pneumoniae be retained separately from the $K$. aerogenes/oxytocaledwardsii group.

The results obtained in our studies are almost identical to those of Bascomb et al. (3). However, we cannot accept that $K$. pneumoniae should be a separate species. The evidence to support such a choice is limited. The names of all four species ( $K$. pneumoniae, $K$. aerogenes, $K$. oxytoca, and $K$. edwardsii) should be considered synonymous, and the designation $K$. pneumoniae (Schroeter) Trevisan 1887 should be used. However, Jain et al. (37) showed by DNA-DNA hybridization that the Oxytocum strains represent a distinct DNA homology from that of $K$. pneumoniae and they proposed a new genus of Enterobacteriaceae with one species for this group. Further study of $K$. oxytoca is required.

The high phenetic resemblance between Klebsiella pneumoniae and Enterobacter aerogenes is apparent from this analysis as well as 
from the earlier study. Only two characteristics, motility and the presence of ornithine decarboxylase, definitely separate the two species, although the production of urease may also be helpful. Strains may occur which may be atypical in one or both of these characteristics. From the results of DNA relatedness studies, Brenner et al. (6) suggested that the genus Klebsiella could be expanded to include $E$. aerogenes strains. Matsumoto (51) demonstrated a close relationship between $K$. pneumoniae and $E$. aerogenes by recombination of chromosomal genes. Bascomb et al. (3) proposed the name Klebsiella mobilis for the organisms known as $E$. aerogenes.

Genus Erwinia. The present studies have not provided any help towards settling the taxonomic conundrum presented by the genus $E r$ winia. It would appear from the literature that three groups exist, corresponding to those described by Dye (16-19) and Lelliot (47). A fourth group of atypical erwiniae (19) has disappeared through reallocation of the strains to other already existing genera $(5,10)$. The three groups correspond to what are sometimes called the "true" Erwinia or "amylovora" group, i.e., those species which cause wilts, galls, or dry necrosis of plants; the "carotovora" group, corresponding to Waldee's Pectobacterium (66), or soft-rot organisms; and the herbicola-lathyri group, or Enterobacter agglomerans (24-26).

Evidence from phenotypic analyses and DNA-DNA homology studies $(5,7,30)$ divides the amylovora group into six species and the carotovora group into five species. The agreement between the two approaches is extremely good, and the consensus of opinion is for retaining the two groups within the family Enterobacteriaceae, but probably as two separate genera, Erwinia and Pectobacterium, respectively.

The third group consists of yellow-pigmented strains, which are more and more frequently being isolated in clinical situations. These strains are of doubtful phytopathogenicity and have, in the past, been assigned a variety of names. Dye (18) and Lelliot (47) subdivided the group into three species, Erwinia herbicola (with two subspecies, subsp. herbicola and subsp. ananas), Erwinia uredovora, and Erwinia stuartii. However, Ewing and Fife (2426) grouped all three species into a single species which they placed in the genus Enterobacter as $E$. agglomerans. Nevertheless, they found it necessary to subdivide $E$. agglomerans into 11 biogroups on the basis of reduction of nitrate, Voges-Proskauer, and indole reactions. Ewing and Fife (24) argued that many of the characteristics of these strains are uniform, and thus they can be included in a single spe- cies. However, examination of their tabulated results presents a somewhat different picture. There is significant biochemical variability within this group, with no justification for creation of a species "ragbag" for purposes of assigning nomenclature to an otherwise unidentified isolate. It also appears that Ewing and Fife are not entirely convinced that the species $E$. agglomerans will not have to be divided at a later date or conceivably that a new genus will not have to be created for these strains. In citing some unpublished data, Brenner et al. (5) indicated that, on the basis of DNA-DNA homology studies, at least 10 different hybridization groups exist within the herbicola group. In view of the coincidence between his hybridization divisions and the phenetic divisions of Dye in the amylovora and carotovora groups, one wonders whether there will also be a striking similarity in the herbicola group.

Strains received as Erwinia species or Enterobacter agglomerans in this study exhibited as much biochemical diversity as reported by other workers. Figures 1 and 2 indicate that seven strains did form a cluster (designated $E$. agglomerans) at about $78 \%$ similarity. However, when the number of strains in the analysis was increased (Fig. 3), this cluster was not robust, and the strains were observed to be more loosely aggregated. On the basis of data for 20 strains of Erwinia, taxonomic recommendations may not be warranted except that very little justification is provided for Enterobacter agglomerans to be a species group as it is presently defined.

In conclusion, some useful observations concerning the taxonomy of several species and genera of the Enterobacteriaceae have been provided by this study. It is hoped that continuing investigations will further clarify those taxonomic issues which are as yet unresolved.

\section{ACKNOWLEDGMENTS}

This work was supported by National Science Foundation grant BMS-72-02227-A03.

The raw data have been submitted to the World Data Bank, Brisbane, Australia, and also are available from the authors upon request.

\section{REPRINT REQUESTS}

Address reprint requests to: Dr. R. R. Colwell, Dept. of Microbiology, University of Maryland, College Park, Md. 20742.

\section{LITERATURE CITED}

1. d'Alessandro, G., and R. Comes. 1956. Sul meccanismo della reazione dell aciclo $\beta$-fenil-propionico negli enterobatteri. Boll. Ist. Sieroter. Milan. 35:202-213.

2. Baird-Parker, A. C. 1963. A classification of micrococci and staphylococci based on physiological and biochemical tests. J. Gen. Microbiol. 30:409-427.

3. Bascomb, S., S. P. Lapage, W. R. Willcox, and M. A. 
Curtis. 1971. Numerical classification of the tribe Klebsiellae. J. Gen Microbiol. 66:279-295.

4. Benton, A. G. 1935. Chitinovorous bacteria. A preliminary survey. J. Bacteriol. 29:449-465.

5. Brenner, D. J., G. R. Fanning, and A. G. Steigerwalt. 1974. Deoxyribonucleic acid relatedness among erwiniae and other Enterobacteriaceae: the gall, wilt, and dry-necrosis organisms (genus Erwinia Winslow et al., sensu stricto). Int. J. Syst. Bacteriol. 24:197204.

6. Brenner, D. J., A. G. Steigerwalt, and G. R. Fanning. 1972. Differentiation of Enterobacter aerogenes from klebsiellae by deoxyribonucleic acid reassociation. Int. J. Syst. Bacteriol. 22:193-200.

7. Brenner, D. J., A. G. Steigerwalt, G. V. Miklos, and G. R. Fanning. 1973. Deoxyribonucleic acid relatedness among erwiniae and other Enterobacteriaceae. I. The soft-rot organisms (genus Pectobacterium Waldee). Int. J. Syst. Bacteriol. 23:205-216.

8. Burkey, L. A. 1928. The fermentation of cornstalks and their constituents. I. Studies on the pectin-fermenting bacteria. Iowa State Coll. J. Sci. 3:57-100.

9. Christensen, W. B. 1949. Hydrogen sulphide production and citrate utilization in the differentiation of the enteric pathogens and the coliform bacteria. Res. Bull. Weld County Health Dept. 1:3.

10. Colwell, R. R., R. Johnson, L. Wan, T. E. Lovelace, and D. J. Brenner. 1974. Numerical taxonomy and deoxyribonucleic acid reassociation in the taxonomy of some gram-negative fermentative bacteria. Int. J. Syst. Bacteriol. 24:422-433.

11. Colwell, R. R., and W. J. Wiebe. 1970. "Core" characteristics for use in classifying aerobic, heterotrophic bacteria by numerical taxonomy. Bull. Ga. Acad. Sci. 18:165-185.

12. Cowan, S. T., K. J. Steel, C. Shaw, and J. P. Duguid. 1960. A classification of the Klebsiella group. J. Gen. Microbiol. 23:601-612.

13. Crosa, J. H., A. G. Steigerwalt, G. R. Fanning, and D. J. Brenner. 1974. Polynucleotide sequence divergence in the genus Citrobacter. J. Gen. Microbiol. 83:271282.

14. Darland, G., and B. R. Davis. 1973. Biochemical and serological characterization of hydrogen sulfide positive variants of Escherichia coli. Center for Disease Control, Atlanta, Ga.

15. Davis, B. R., and W. H. Ewing. 1966. The biochemical reactions of Citrobacter freundii. Center for Disease Control, Atlanta, Ga.

16. Dye, D. 1968. A taxonomic study of the genus Erwinia. I. The "Amylovora" group. N. Z. J. Sci. 11:590-607.

17. Dye, D. 1969. A taxonomic study of the genus Erwinia. II. The "Carotovora" group. N. Z. J. Sci. 12:81-97.

18. Dye, D. 1969. A taxonomic study of the genus Erwinia. III. The "Herbicola" group. N. Z. J. Sci. 12:223-236.

19. Dye, D. 1969. A taxonomic study of the genus Erwinia. IV. "Atypical" erwiniae. N. Z. J. Sci. 12:833-838.

20. Edwards, P. R., and W. H. Ewing. 1972. Identification of Enterobacteriaceae, p. 1-362. Burgess Publishing Co., Minneapolis.

21. Ewing, W. H. 1971. Biochemical characterization of Citrobacter freundii and Citrobacter diversus. Center for Disease Control, Atlanta, Ga.

22. Ewing, W. H., and B. R. Davis. 1972. Biochemical characterization of Citrobacter diversus (Burkey) Werkman and Gillen and designation of the neotype strain. Int. J. Syst. Bacteriol. 22:12-18.

23. Ewing, W. H., B. R. Davis, and R. W. Reavis. 1957. Phenylalanine and malonate media and their use in enteric bacteriology. Publ. Health Lab. 15:153-167.

24. Ewing, W. H., and M. A. Fife. 1972. Enterobacter ag. glomerans (Beijerinck) comb. nov. (the HerbicolaLathyri bacteria). Int. J. Syst. Bacteriol. 22:4-11.
25. Ewing, W. H., and M. A. Fife. 1972. Enterobacter agglomerans. The herbicola-lathyri bacteria. Center for Disease Control, Atlanta, Ga.

26. Ewing, W. H., and M. A. Fife. 1972. Biochemical characterization of Enterobacter agglomerans. Center for Disease Control, Atlanta, $\mathrm{Ga}$.

27. Fred, E. B., I. L. Baldwin, and E. McCoy. 1932. Root nodule bacteria and leguminous plants. Univ. Wis. Stud. Sci. 5:1-343.

28. Frederiksen, W. 1970. Citrobacter koseri (n.sp.), a new species within the genus Citrobacter with a comment on the taxonomic position of Citrobacter intermedium (Werkmann and Gillen). Publ. Fac. Sci. Univ. J. E. Purkyne Brno. 47:89-94.

29. Gaby, W. L., and E. Free. 1958. Differential diagnosis of Pseudomonas-like microorganisms in the clinical laboratory. J. Bacteriol. 76:442-444.

30. Gardner, J. M., and C. I. Kado. 1972. Comparative base sequence homologies of the deoxyribonucleic acids of Erwinia species and other Enterobacteriaceae. Int. J. Syst. Bacteriol. 22:201-209.

31. Gordon, R. E., and J. M. Mihm. 1957. A comparative study of some strains received as nocardiae. J. Bacteriol. 73:15-27.

32. Gordon, R. E., and M. M. Smith. 1955. Rapidly growing, acid fast bacteria. II. Species description of $\mathrm{Myco}$ bacterium fortuitum Cruz. J. Bacteriol. 69:502-507.

33. Gross, R. J., and B. Rowe. 1974. The serology of Citrobacter koseri, Levinea malonatica and Levinea amalonatica. J. Med. Microbiol. 7:155-161.

34. Haynes, W. C. 1951. Pseudomonas aeruginosa-its characterization and identification. J. Gen. Microbiol. 5:939-950.

35. Hinshaw, W. R. 1941. Cysteine and related compounds for differentiating members of the genus Salmoklla. Hilgardia 13:583-621.

36. Hugo, W. B., and E. G. Beveridge. 1962. A quantitative and qualitative study of the lipolytic activity of single strains of seven bacterial species. J. Appl. Bacteriol. 25:72-82.

37. Jain, K., K. Radsak, and W. Mannheim. 1974. Differentiation of the Oxytocum group from Klebsiella by deoxyribonucleic acid-deoxyribonucleic acid hybridization. Int. J. Syst. Bacteriol. 24:402-407.

38. Johnson, R., R. R. Colwell, R. Sakazaki, and K. Tamura. 1975. Numerical taxonomy study of the Enterobacteriaceae. Int. J. Syst. Bacteriol. 25:12-37.

39. Kaluzewski, S. 1967. Taxonomic position of indole-positive strains of Klebsiella. Exp. Med. Microbiol. $19: 350-359$.

40. Kauffmann, F., and A. Petersen. 1956. The biochemical group and type differentiation of Enterobacteriaceae by organic acids. Acta Pathol. Microbiol. Scand. 38:481-491.

41. King, E. O., M. K. Ward, and D. E. Raney. 1954. Two simple media for the demonstration of pyocyanin and fluorescin. J. Lab. Clin. Med. 44:301-307.

42. Kohn, J. 1955. A preliminary report of a new gelatin liquefaction method. J. Clin. Pathol. 6:249.

43. Koser, S. A. 1923. Utilization of the salts of organic acids by the colon-aerogenes group. J. Bacteriol. 8:493-520.

44. Kovacs, N. 1956. Identification of Pseudomonas pyocyanea by the oxidase reaction. Nature (London) 178:703.

45. Lautrop, H. 1956. Gelatin liquefying Klebsiella strains (Bacterium oxytocum (Flügge)). Acta Pathol. Microbiol. Scand. 39:375-384.

46. Lautrop, H., I. Orskov, and K. Gaarslev. 1971. Hydrogen sulphide producing variants of Escherichia coli. Acta Pathol. Microbiol. Scand. Sect. B 79:641-650.

47. Lelliot, R. A. 1974. The genus Erwinia, p. 1-1246. In R. E. Buchanan and N. E. Gibbons (ed.), Bergey's man- 
ual of determinative bacteriology, 8 th ed. Williams and Wilkins Co., Baltimore.

48. Le Minor, L., and F. Ben Hamida. 1962. Avantages de la recherche de la $\beta$-galactosidase sur celle de la fermentation du lactose en milieu complexe dans le diagnostic bacteriologique en particulier des Enterobacteriaceae. Ann. Inst. Pasteur Paris 102:267-277.

49. Macfarlane, R. G., C. L. Oakley, and C. G. Anderson. 1941. Haemolysis and the production of opalescence in serum and lecithovitellin by the $\alpha$-toxin of Clostridium welchii. J. Pathol. Bacteriol. 52:99-103.

50. Macierewicz, M. 1966. A proposal of a new group (genus) of Enterobacteriaceae. Med. Dosw. I. Mikrobiol. 18:333-339.

51. Matsumoto, H. 1973. Genetic recombination between Klebsiella pneumoniae and Enterobacter aerogenes. Genet. Res. 21:47-55.

52. Niven, C. F., K. L. Smiley, and J. M. Sherman. 1942. The hydrolysis of arginine by streptococci. J. Bacteriol. 43:651-660.

53. Orskov, I. 1957. Biochemical types in the Klebsiella group. Acta Pathol. Microbiol. Scand. 40:155-162.

54. Orskov, I., and F. Orskov. 1973. Plasmid-determined $\mathrm{H}_{2} \mathrm{~S}$ character in Escherichia coli and its relation to plasmid-carried raffinose fermentation and tetracycline resistance characters. J. Gen. Microbiol. 77:487499.

55. Richard, C., B. Brisou, and J. Lioult. 1972. Etude taxonomique de "Levinea" nouveau genre de la famille des Entérobactéries. Ann. Inst. Pasteur Paris. 122:1137-1146.

56. Rustigan, R., and C. A. Stuart. 1941. Decomposition of urea by Proteus. Proc. Soc. Exp. Biol. Med. 47:108112.

57. Sedlak, J. 1973. Present knowledge and aspects of Citrobacter. Curr. Top. Microbiol. Immunol. 62:41-59.

58. Sedlak, J. 1974. The genus Citrobacter, p. 1-1246. In
R. E. Buchanan and N. E. Gibbons (ed.), Bergey's manual of determinative bacteriology, 8th ed. Williams and Wilkins Co., Baltimore.

59. Sierra, G. 1957. A simple method for the detection of lipolytic activity of microorganisms and some observations on the influence of the contact between cells and fatty substrates. Antonie van Leeuwenhoek $\mathrm{J}$. Microbiol. Serol. 23:15-22.

60. Simmons, J. S. 1926. A culture medium for differentiating organisms of the typhoid-colon aerogenes groups and for isolation of certain fungi. J. Infect. Dis. 39:209-214.

61. Singer, J., and B. E. Volcani. 1955. An improved ferric chloride test for differentiating Proteus-Providence group from other Enterobacteriaceae. J. Bacteriol. 69:303-306.

62. Sneath, P. H. A., and R. R. Sokal. 1973. Numerical taxonomy: the principles and practice of numerical classification, p. 1-573. W. H. Freeman, San Francisco.

63. Starr, M. P. 1947. Causal agent of bacterial root and stem disease of guayule. Phytopathology 37:291.

64. Tittsler, R. P., and L. A. Sandholzer. 1935. Studies on the Escherichia-Aerobacter intermediates. J. Bacteriol. 29:349-361.

65. Vaughn, R. H., and M. Levine. 1942. Differentiation of the "intermediate" coli-like bacteria. J. Bacteriol. 44:487-505.

66. Waldee, E. L. 1945. Comparative studies of some peritrichous phytopathogenic bacteria. Iowa State Coll. J. Sci. 19:435-484.

67. Werkman, C. H., and G. F. Gillen. 1932. Bacteria producing trimethylene glycol. J. Bacteriol. 23:167-182.

68. Young, V. M., D. M. Kenton, B. J. Hobbs, and M. R. Moody. 1971. Levinea, a new genus of the family Enterobacteriaceae. Int. J. Syst. Bacteriol. 21:58-63. 